\title{
Dynamics of coupled maps with a conservation law
}

\author{
R. O. Grigoriev and M. C. Cross \\ Condensed Matter Physics 114-36, California Institute of Technology, Pasadena, California 91125
}

(Received 19 August 1996; accepted for publication 21 January 1997)

\begin{abstract}
A particularly simple model belonging to a wide class of coupled maps which obey a local conservation law is studied. The phase structure of the system and the types of the phase transitions are determined. It is argued that the structure of the phase diagram is robust with respect to mild violations of the conservation law. Critical exponents possibly determining a new universality class are calculated for a set of independent order parameters. Numerical evidence is produced suggesting that the singularity in the density of Lyapunov exponents at $\lambda=0$ is a reflection of the singularity in the density of Fourier modes (a "Van Hove" singularity) and disappears if the conservation law is broken. Applicability of the Lyapunov dimension to the description of spatiotemporal chaos in a system with a conservation law is discussed. (C) 1997 American Institute of Physics. [S1054-1500(97)00102-X]
\end{abstract}

The essential features of an extended non-equilibrium system can be effectively modeled using a coupled map lattice. Such a model includes particular couplings between lattice sites, and can provide a straightforward method for simulating extended systems with highly nonlinear local interactions. The question addressed in the current study concerns how the behavior of systems subject to certain conservation laws (conserving systems) may differ from non-conserving systems, unconstrained by such laws. The consequence of violating a conservation law is also considered. To this end we have conducted a detailed study of a simple model for both conserving and non-conserving non-equilibrium systems. The observed differences could help in the identification of hidden conservation laws, and mechanisms for their violation, for real physical, chemical and biological systems.

\section{INTRODUCTION}

Coupled map lattices (CML) with an additive conserved quantity became a subject of intensive research recently. ${ }^{1-3}$ On the one hand such CML's are often obtained as phenomenological models representing the dynamics of a large number of interacting macroscopic structures. On the other hand they are a natural result of finite-difference approximations of continuous nonlinear partial differential equations such as the Kuramoto-Sivashinky equation, ${ }^{4}$ or a phenomenological Cahn-Hilliard equation ${ }^{5}$ describing the nonlinear dynamics of several systems with conserved-order-parameter.

Models of this class are expected to represent several typical non-equilibrium physical phenomena. For instance, surface waves, ${ }^{6}$ where the average depth of the fluid in the container is conserved, electrohydrodynamic instabilities in nematics with insulating plates, ${ }^{7}$ where the total charge is conserved, disturbances in the atmosphere and ocean systems, where the total (depth integrated) heat is conserved, and even some types of hard turbulence. ${ }^{8}$ As such they are significant as tools for studying the complex spatiotemporal behavior of spatially extended non-linear systems, especially in the strongly chaotic regime, where the analytical methods designed for weak nonlinearities become ineffective.

For coupled map systems with an additive conserved quantity several major points are still awaiting clarification. First of all, what is the effect of the conservation law on the structure of the phase diagram and the character of the phase transitions? What is the connection between the conservation law and singularities observed in the density of Lyapunov exponents $^{1,9,10}$ and what is the origin and significance of these singularities? Another important issue is to determine which parameters best describe the dynamics of an extended system and what their limitations are. In particular, it is unclear whether the Lyapunov spectrum provides any exclusive information about the chaotic dynamics that cannot be obtained by other methods. And finally, we would like to know whether the reduction of the system dynamics to symbolic form preserves the main characteristics of the chaotic dynamics and can provide us with the complete description of the latter.

The model chosen should be relatively simple yet represent most of the typical features under consideration. Most important, it should have a non-trivial phase diagram. With this in mind we pick the one-dimensional collection of $L$ diffusively coupled chaotic maps:

$$
u_{i}^{n+1}=u_{i}^{n}+\left(f\left(u_{i-1}^{n}\right)-2 f\left(u_{i}^{n}\right)+f\left(u_{i+1}^{n}\right)\right),
$$

with periodic boundary conditions imposed. The local map was chosen to be

$$
f(x)=a x+b z(1-z), \quad z=\operatorname{frac}(x) .
$$

This CML can also be regarded as a finite difference approximation of the differential equation continuous in both space and time,

$$
\partial_{t} u(x, t)=\partial_{x}^{2} f(u(x, t)) .
$$

A differential equation of this form represents the competition between two opposing tendencies: generation of chaotic 
perturbations by the non-linear part of $f(x)$ and dissipation of these perturbations by the diffusive coupling introduced by the second spatial derivative.

The model clearly possesses the conservation law:

$$
u=\frac{1}{L} \sum_{i=1}^{L} u_{i}^{n}=\mathrm{const}
$$

so that aside from the two parameters of the local map $a$ and $b$ we have an additional control parameter-the additive conserved quantity $u$, which is defined by the initial condition and is of critical importance to the behavior of the system.

We are primarily interested in the dynamics in the "thermodynamic limit' $L \rightarrow \infty$, although the numerics is obviously restricted to finite systems.

The outline of this paper is as follows. In section II we study the phase diagram of the coupled map model. In section III we introduce the symbolic (reduced) description of the system dynamics. In section IV we discuss the quantitative description of the reduced dynamics. In section $\mathrm{V}$ we study phase transitions in our model and determine their types. In particular, we study the effect of the conservation law on the type of the transition and on the values of critical exponents. In section VI we discuss the applicability of the Lyapunov dimension to the description of the dynamics in the system. In section VII we present numerical data suggesting the reason for the existence of the singularity in the spectrum of Lyapunov exponents. In section VIII we demonstrate the effect of violations of the conservation law on the system dynamics. The paper ends with a summary and discussion in section IX.

\section{PHASE DIAGRAM}

Despite its simple form, this model has a very rich structure. Numerical simulations show that depending on the values of the control parameters it can be strongly or mildly chaotic, show spatiotemporal intermittency (STI), give rise to pattern formation or simply decay into the spatially uniform stable state, or (for $L$ - even) a 2-cycle in both space and time. Both asymptotically regular (non-chaotic) states can be described by a single equation:

$$
u_{j}^{n}=u+(-1)^{j+n} A .
$$

In order to gain some insight into the phase diagram we analytically determine the boundaries of the stability regions of the two non-chaotic asymptotic states of the system. Linear stability analysis of the spatially uniform state gives Lyapunov exponents (equivalent to the growth rates) in the form

$$
\lambda_{n}=\ln \left|1-4(a+b-2 b u) \sin ^{2}\left(\frac{\pi n}{L}\right)\right| .
$$

From this one can conclude that the region of linear stability of the uniform phase is given by

$$
\frac{a+b}{2 b}-\frac{1}{4 b}<u<\frac{a+b}{2 b} .
$$

Note that at the upper boundary all exponents corresponding to all Fourier modes $k=2 \pi n / L$ become real and positive, whereas at the lower boundary the $k=\pi$ mode goes unstable to a period 2 oscillation.

Stability analysis of the square of the map (1) gives the boundaries of the linear stability region of the 2-cycle state written in a slightly different form:

$$
u_{i}^{n}= \begin{cases}u_{+}, & \text {if } i+n \text { is even, } \\ u_{-}, & \text {if } i+n \text { is odd. }\end{cases}
$$

The dynamics is invariant under the transformation $u \rightarrow u \pm 1$. As a consequence it turns out that there are two types of 2-cycles possible. One (type-I) with

$$
\left[u_{-}\right]=\left[u_{+}\right]-1
$$

$([\cdot]$ denotes the integer part) requires

$$
u_{ \pm}=u\left(1 \pm \frac{2 b}{4 b u+1-2 a}\right)
$$

and has a stability region bounded by the surfaces given by the following two equations:

$$
\left(1-2 a+2 b+4 b u_{-}\right)\left(1-2 a-2 b+4 b u_{+}\right)=-1
$$

and

$$
u=\frac{a}{2 b} \text {. }
$$

At the upper boundary (12) the $k=\pi$ mode becomes growing, while at the lower boundary (11) a Hopf bifurcation of the $|k|=\pi / 2$ modes occurs. This is quite fortunate, since one can obtain the analytic expressions for the phase boundaries for a system of arbitrary size $L$ from the analysis of a system with $L=4$.

The other (type-II) 2-cycle is such that

$$
\frac{u_{-}+u_{+}}{2}=u \text { and }\left[u_{-}\right]=\left[u_{+}\right]
$$

and, for the local map $f(x)$ given by eq. (2), it can only exist at the stability boundary of the uniform state given by

$$
u=\frac{a+b}{2 b}-\frac{1}{4 b}
$$

but can have an arbitrary amplitude $A=\left(u_{+}-u_{-}\right) / 2$, subject only to the condition (13).

Figure 1 presents two cross sections of the parameter space. We will denote the region where the uniform state (1-cycle) is linearly stable as the phase L1. Similarly, the phase L2 will stand for the linear stability region of the 2-cycle state. As we are going to see later, the 2-cycle state is not the only possible asymptotic state in this phase, so it will be useful to introduce the additional subdivision of this phase into subphases for a more detailed analysis.

The attractors of the phases $\mathrm{T} 1$ and $\mathrm{T} 2$ are chaotic. The two phases are not essentially different. One can easily find a continuous trajectory in the parameter space that would join arbitrary points in phase $\mathrm{T} 1$ with those in phase $\mathrm{T} 2$ without intersecting any phase boundary. Although the dynamics of 


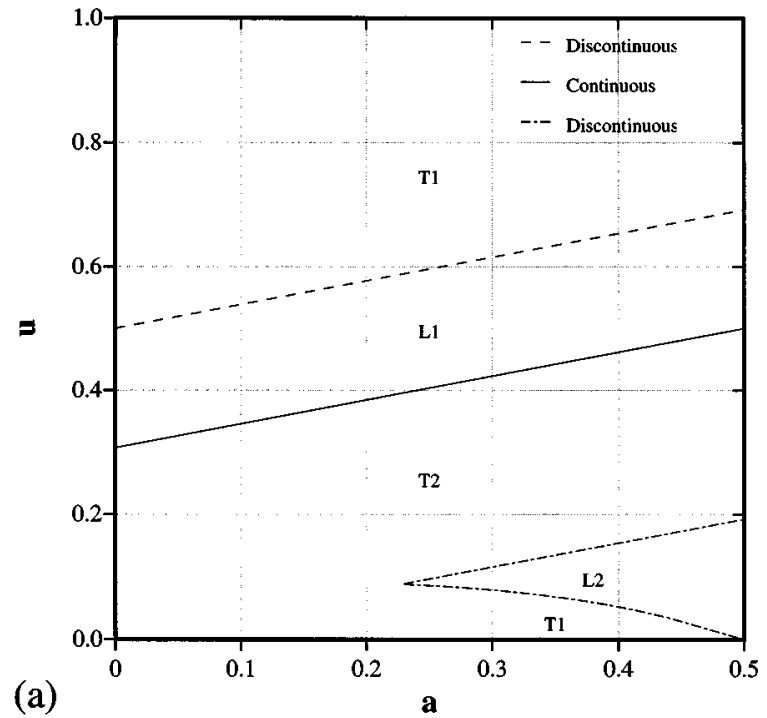

(a)

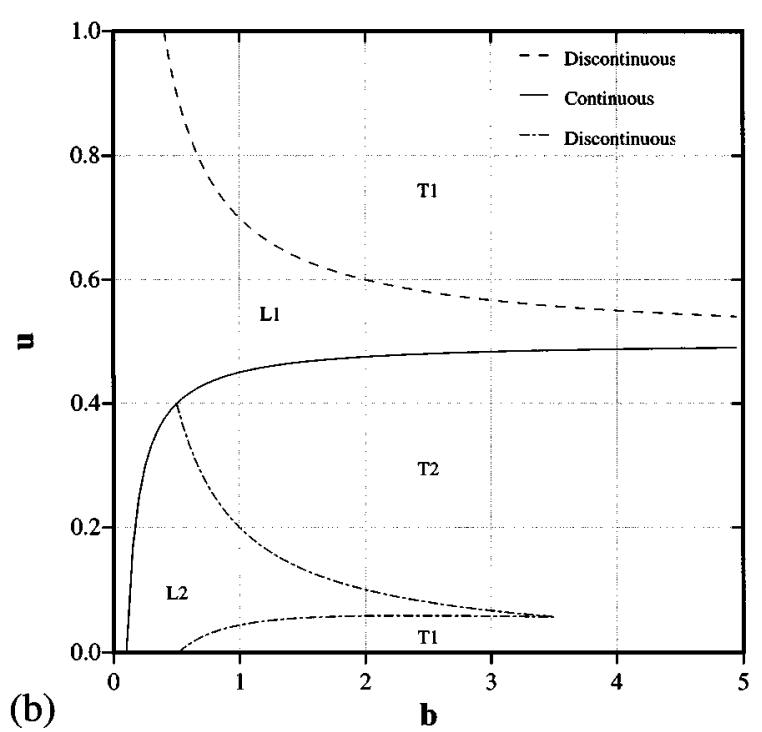

FIG. 1. Cross-sections of the phase diagram in a three-dimensional parameter space (a) $u$ vs $a$ at $b=1.3$; (b) $u$ vs $b$ at $a=0.4$. The solid line corresponds to a continuous phase transition. The dashed and the dotted lines denote the phase boundaries where discontinuous phase transitions between chaotic and non-chaotic states occur.

the system is somewhat different in the two phases for the set of parameters used, this distinction is introduced mostly for convenience.

An important property of the system is that despite the large number of degrees of freedom the type of the attractor (and therefore, the type of behavior, if one excludes long transients) seems to be uniquely determined by the values of control parameters $a, b$ and $u$ and is independent of the details of the initial state. If there exists a single attractor, then the basin of attraction is (almost) all configuration space. In this case averages over the attractor can be estimated as the time average from a single initial condition, and we can call the system ergodic. (We assume that, at least with respect to the numerical computation, there exists a "physical measure" such as discussed by Eckmann and
Ruell ${ }^{11}$ that eliminates any ambiguity in the choice of invariant measures on the attractor.)

Indeed, numerical data suggest that the attractor is unique in most of the parameter space. However it is not always the case: close to the boundary L1-T1 a frozen pattern may form and the details of a pattern do depend on the initial conditions. So, on the timescale used in our calculations (typically of order 1 million iterations) the system did not appear ergodic. Of course, that does not mean that the system could not become ergodic on a yet larger timescale.

Another exception is the regions inside the ordered phases L1 and L2, where two attractors, chaotic and nonchaotic, can coexist. We will later discuss this situation in more detail.

One of the objectives of this paper is to study the effect of the conservation law on the dynamics of the extended chaotic system. We therefore would like to follow the changes in the parameters characterizing the dynamics as a function of the conserved quantity $u$. In further work we will fix the values of the other two parameters at $a=0.4$, $b=1.3$, which (as seen from the phase diagram, fig. 1) will allow us to study the regimes of interest (various chaotic as well as periodic states).

Looking at the phase diagram, one can expect that this model should experience at least four bifurcations or phase transitions as $u$ is varied in the interval $0<u<1$. (Since we are interested in diverging correlation lengths near the transitions between different states in the $L \rightarrow \infty$ limit, and the consequent possibility of universal exponents, we will use the term "phase transitions" rather than "bifurcations.") Equations (11) and (12) give us $u_{a} \approx 0.0520$ and $u_{b}=u_{d}-0.5 \approx 0.1538$ as the boundaries of the 2-cycle stability region. According to eq. (7) the uniform state loses its stability at $u_{c} \approx 0.4615$ and $u_{d} \approx 0.6538$.

Phase transitions from ordered to chaotic states are common occurrences in coupled map lattices. ${ }^{12}$ They may be continuous or discontinuous. For continuous transitions we might expect the transitions to fall into various universality classes, characterized by scaling exponents for various diagnostics of the chaos near the transition. In this case the transitions in CML's may be representative of transitions to spatiotemporal chaos in more general extended non-equilibrium systems. Although the qualitative features determining the universality classes are not understood one expects that symmetries, such as the Ising symmetry studied by Miller and Huse, ${ }^{13}$ and conservation laws, rather than the detailed properties of the local maps, will be important.

In particular, it has been suggested ${ }^{14}$ that under certain very general conditions (e.g., in systems with a unique absorbing state) the transition should fall into the universality class of directed percolation, although some counter examples to this statement are known. It is nevertheless interesting to check whether any of the phase transitions in our model belong to the universality class of directed percolation, since, as we are going to see below, the absorbing state in our model is in fact unique for any choice of control parameters. 


\section{REDUCED DYNAMICS}

In order to understand the spatial dynamics of the system better and to see the finer details of the phase diagram we will (following Kaneko ${ }^{15}$ ) reduce the description of the dynamics to a finite number of states: in terms of this reduced dynamics each site of the lattice can be marked either "laminar" or "turbulent" thus making up a set of laminar and turbulent domains. Then one would naively expect a laminar domain to be a region of the lattice with a relatively slow chaotic dynamics (no large, if any, positive local Lyapunov exponents ${ }^{16}$ ); and a turbulent domain to be a region where the chaotic dynamics is fast (with at least a few large positive local Lyapunov exponents). Positive Lyapunov exponents will inevitably make the turbulent domains spatially irregular, while laminar domains tend to be spatially regular.

We will not put the terms "laminar" and "turbulent" in quotes below, nevertheless one should clearly understand that these are just a convenient notation and thus are restricted in meaning.

We need a simple criterion that will determine whether a given site belongs to a turbulent or laminar domain (we will only consider as laminar states those that are close to either uniform or 2-cycle configurations). The simple way to distinguish between (uniform) laminar and turbulent sites numerically would be to call a site $j$ laminar on time step $n$ if

$$
\left|u_{j-1}^{n}-u_{j}^{n}\right|<\epsilon \text { and }\left|u_{j}^{n}-u_{j+1}^{n}\right|<\epsilon,
$$

and turbulent otherwise. The problem with this definition is that any 2-cycle with amplitude,

$$
A_{j}^{n}=\frac{\left|u_{j-1}^{n}-u_{j}^{n}\right|}{2}>\epsilon,
$$

would be considered turbulent, which is clearly incorrect. Therefore, the definition of a laminar domain has to be generalized to include a zig-zag pattern with a slowly varying envelope of arbitrary amplitude:

$$
\frac{\left|A_{j}^{n}-A_{j+1}^{n}\right|}{\left|A_{j}^{n}+A_{j+1}^{n}\right|}<\epsilon .
$$

In this particular model we set $\epsilon \sim 0.01$.

Based on this reduction one can distinguish between various types of dynamics in the system. Pictures representing the spatiotemporal evolution of the system in symbolic form are so characteristic that one can easily determine which part of phase diagram the system is in just by looking at the patterns. We will examine several typical pictures, highlighting the most interesting phenomena observed in this model.

The behavior of our CML in the phase L2 is non-trivial. We already know that inside the phase there is a stable ordered state. Our numerical data imply however that this ordered 2-cycle state is stable in the non-linear sense only for values of u satisfying $u_{a}<u_{p}<u<u_{n}<u_{b}$ (figure 2). As we are going to see later, it is quite hard to determine the critical values $u_{p}$ and $u_{n}$ exactly, but they seem to approach the fixed values $u_{p} \approx 0.063$ and $u_{n} \approx 0.082$ in the thermodynamic limit $L \rightarrow \infty$.

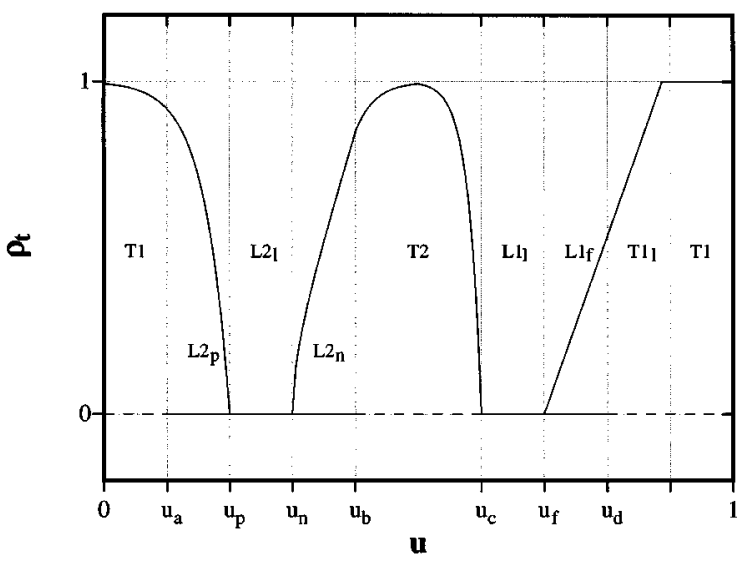

FIG. 2. One-dimensional cross-section of the phase diagram. Measure of the set of turbulent domains, $\rho_{t}$, is plotted schematically (actual numeric results are presented in fig. 9) as a function of parameter $u$. Solid lines correspond to the values for the linearly stable asymptotic states and dashed lines correspond to the unstable asymptotic states. Two different linearly stable states coexist in the sub-phases $L 2_{p}, L 2_{n}, L 1_{f}$. A locked chaotic state always forms in $T 1_{l}$ and for some initial states in $L 1_{f}$ as well.

For $u_{p}<u<u_{n}$ the ordered state is reached through a chaotic transient whose lifetime is usually very small, no matter what initial condition is chosen, and thus the asymptotic behavior dominates. For relatively small systems $(L \lesssim 400)$ this results in a fast decay of any initial state into a limit 2-cycle. For larger systems the quiescent asymptotic state may be completely regular as well, but it may also have a few localized turbulent defects moving with unit speed through the homogeneous 2-cycle background. Defects moving in opposite directions eventually die out, colliding with each other. Similar properties were observed in a number of one- and two-dimensional models featuring spatiotemporally intermittent dynamics (see Ref. 17 and references therein for example).

For $u_{a}<u<u_{p}$ as well as for $u_{n}<u<u_{b}$ the ordered state is only conditionally stable (stable to small perturbations) and most initial conditions result in a spatiotemporally chaotic asymptotic state consisting of a combination of laminar and turbulent domains, with the laminar domains featuring exactly the same structure as the ordered state: the type-I 2-cycle. Therefore the phase L2 can be subdivided into three sub-phases according to whether any turbulent domains are present in the asymptotic state together with the laminar background whose structure is the same throughout the phase L2.

Figure 2 shows schematically the (time averaged) fraction of the lattice occupied by turbulent domains in the phase L2 as a function of parameter $u$. In the sub-phase $L 2_{l}$ $\left(u_{p}<u<u_{n}\right)$ the asymptotic state is laminar. In the subphases $L 2_{p} \quad\left(u_{a}<u<u_{p}\right)$ and $L 2_{n} \quad\left(u_{n}<u<u_{b}\right)$ the asymptotic state can be either laminar or spatiotemporally chaotic. It is interesting to note that the characteristic patterns produced by the turbulent domains are different in the two sub-phases featuring a persistent spatiotemporally intermittent state. As a result one might expect to see two quite different phase transitions at $u=u_{p}$ and $u=u_{n}$. 

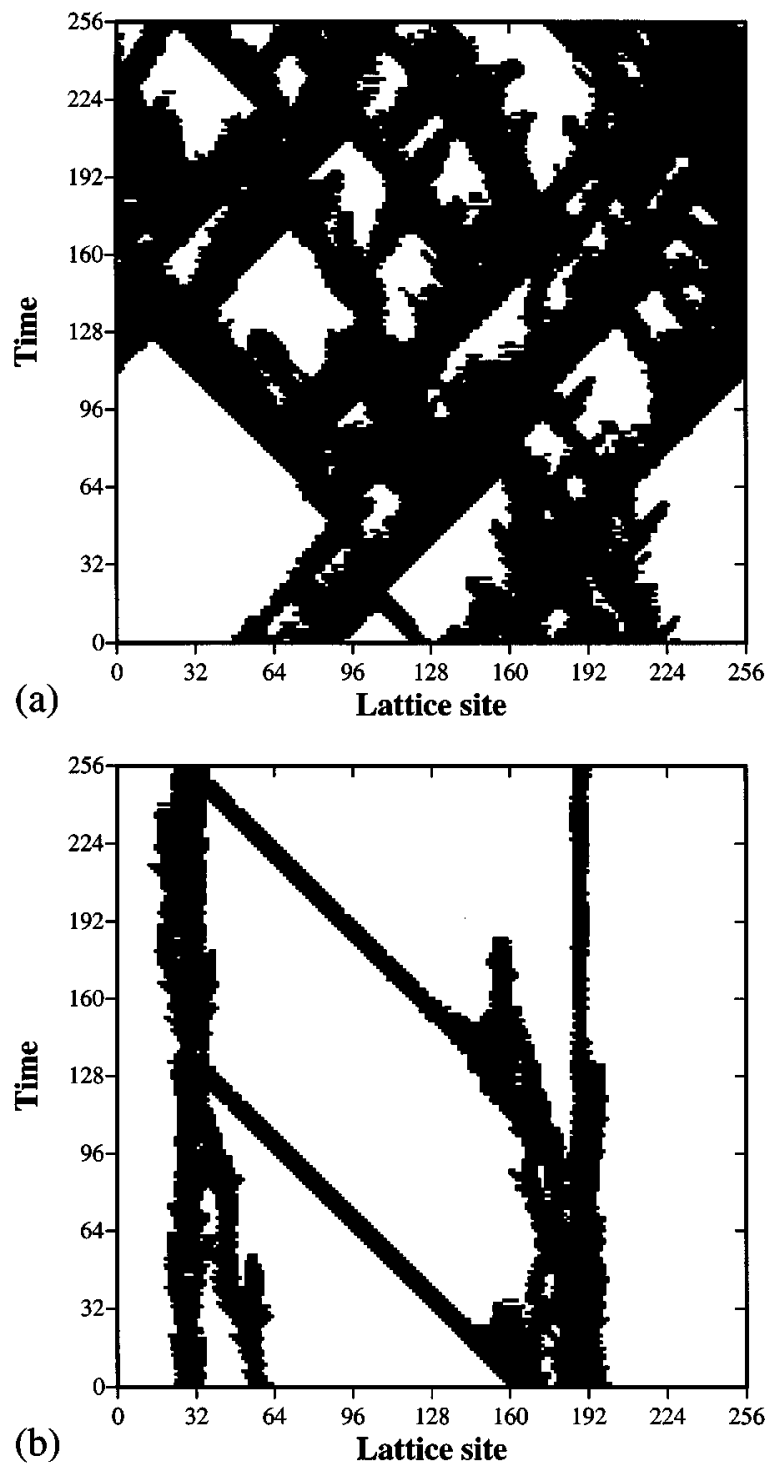
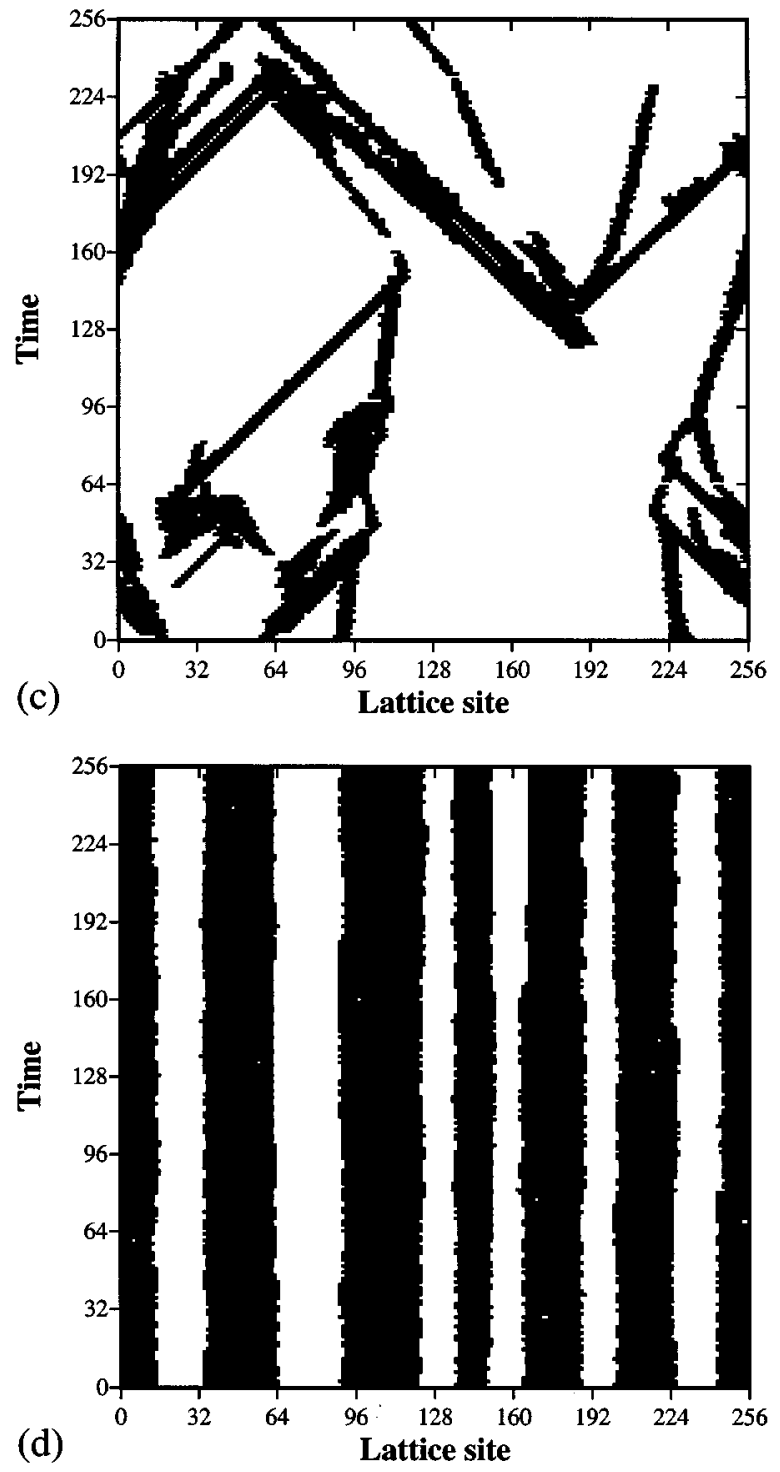

FIG. 3. Symbolic representation of the system dynamics close to the phase transition points: turbulent sites are marked black and laminar sites are white. (a) "Percolating", state at $u=0.06$; (b) "nuclear'" state at $u=0.09$; (c) defect dominated state at $u=0.46$; (d) frozen pattern at $u=0.66$. Lattice size is 256 .

A pattern typical for the sub-phase $L 2_{p}$ is presented on fig. 3(a). This state is very similar to some of the spatiotemporally intermittent states of a (1-dimensional) model studied by Chate and Manneville. ${ }^{19}$ The major difference is that in our model turbulent domains typically have a larger size. Since the absorbing (laminar) state is an ordered one and is unique, we might expect a phase transition at $u_{p}$ to belong to the universality class of directed percolation. ${ }^{18}$

In fact, this kind of STI state is not specific to discrete extended systems. A very similar state can be observed in some continuous models as well, for instance, ${ }^{20}$ in a damped Kuramoto-Sivashinsky equation:

$$
\partial_{t} u(x, t)=-\eta u-\partial_{x}^{2} u-\partial_{x}^{4} u-u \partial_{x} u,
$$

with $\eta \approx 0.075$.

Figure 3(b) presents another type of spatiotemporally intermittent state that can be observed in the phase L2. Its distinguishing feature is that it is composed of a set of vir- tually immobile turbulent nuclei "'mediated"' by creation and absorption of non-linear waves, propagating trough the laminar background with unit velocity. This type of "nuclear'" STI state is characteristic for the sub-phase $L 2_{n}$.

The phase L1 also features an internal structure (see fig. 2). For $u_{c}<u<u_{f}$ (sub-phase $L 1_{l}$ ) any initial configuration decays quickly into a uniform stable state. In other words, the uniform state is non-linearly stable. For $u_{f}<u<u_{d}$ (subphase $L 1_{f}$ ) the uniform state is only conditionally stable and large deviations from it result in a spatiotemporally chaotic asymptotic state. The transition point separating the two subphases is estimated to be $u_{f} \approx 0.53$ in the thermodynamic limit.

Figure 3(d) represents a frozen pattern, characteristic of the chaotic asymptotic state of the system in the sub-phase $L 1_{f}$ and in the phase T1 close to the boundary with L1 (we will denote this region $T 1_{l}$ ). It is probably more appropriate to call this type of dynamics locked chaos: the chaotic state 
is almost stationary in terms of reduced dynamics, with chaos localized in turbulent domains. As was briefly mentioned in section II, the dimensions and locations of the turbulent domains depend on the details of the initial state of the system. The laminar state in this sub-phase is uniform.

And finally, fig. 3(c) gives the reduced dynamics representation of critical behavior displayed by the CML in the chaotic phase T2 close to the boundary with L1. Here we encounter yet another example of a spatiotemporally intermittent state observed in our model. The laminar state is again a 2-cycle (more specifically the type-II 2-cycle) and it is also absorbing, i.e., a new turbulent domain (we will call these defects because of their small size) can never originate inside a laminar domain, it can only be spawned by other defect(s). However a defect can be consumed by a laminar domain, or alternatively it can be destroyed in a collision with another defect.

The most prominent feature of this picture is "spontaneous" creation and annihilation of turbulent pulses (defects) moving in different directions with different (but constant) velocities. Therefore we may alternatively regard these defects as traveling waves. Naively one would expect that the condition $\xi \gg 1$, where $\xi$ is the correlation length, is necessary and might also be sufficient for the formation of a number of traveling waves. Numerical results for our model support this assumption (in disagreement with the stronger restriction, ${ }^{21}$ according to which the correlation length $\xi$ should be comparable to the size of the system $L$ ).

Nevertheless, since in the strongly chaotic regime the correlation length is of order one lattice spacing, the condition $\xi \gg 1$ is usually only satisfied close to the hypersurfaces in the parameter space on which the correlation length diverges, i.e., where a continuous phase transition occurs. This is clearly the case of fig. 3(c): we have a continuous phase transition at $u=u_{c} \approx 0.4615$.

Deeper in the chaotic phases T1 and T2, away from the phase boundaries, strongly chaotic behavior could be observed. Here almost all sites on the lattice exhibit turbulent behavior, and only occasionally a laminar domain of a very small size is created and then quickly consumed by the neighboring turbulent sites. We would call this type of dynamics strong chaos in contrast to the mild chaos, where all chaotic dynamics is localized to turbulent domains, occupying only a part of the lattice, while the rest of it is in the laminar state.

\section{DOMAIN LENGTHS}

A quantitative description of the reduced dynamics is provided by the probability distribution functions $P_{t}(l)$ and $P_{l}(l)$ giving the probability for a turbulent (laminar) domain to have length $l$. In order to calculate these functions numerically we used a single random initial condition and let the system evolve, counting how many times a laminar (turbulent) domain of a given size formed. The resulting distributions did not depend on a particular choice of the initial condition for any given set of control parameters corresponding to ergodic dynamics, i.e., everywhere except the subphase $L 1_{f}$.

Our calculations show that in the chaotic state (away from phase boundaries) both $P_{t}(l)$ and $P_{l}(l)$ decay exponentially. Even more important, typical lengthscales appear to be almost independent of the value of the conserved quantity $u$, being $l_{l} \approx 1$ for laminar and $l_{t} \sim 10^{3}$ for turbulent regions. This is consistent with the point of view that most of the system is in a turbulent state and the probability of encountering a laminar region at any particular location is very small (but finite and independent of the system size) and decreases rapidly with increasing domain length, while there is no spatial structures defining alternative lengthscales.

The behavior of length distribution functions might be expected to change substantially as the system gets close to the phase transition points. For example, if the transition is continuous the correlation length may grow and the critical effects might introduce alternative lengthscales that would modify the form of the distribution functions.

We first examine the "percolating" STI state characteristic of the sub-phase $L 2_{p}$. Figure 4(a) shows that both $P_{t}(l)$ and $P_{l}(l)$ decay exponentially for large $l$. This is compatible with the assumption that the phase transition at $u=u_{p}$ might in fact belong to the universality class of directed percolation. ${ }^{18}$ It is interesting to note though, that $P_{l}$ has two branches, one corresponding to even, the other to odd size of a domain. About the only useful information that one can extract from this data is typical lengthscales of laminar and turbulent domains, which, for $u=0.06$, appear to be tens of lattice spacings for both.

In the previous section we saw that the STI states observed inside $L 2_{p}$ and $L 2_{n}$ are considerably different. As a result fig. 4(b), which corresponds to the "nuclear" state, differs from fig. 4(a) substantially: though $P_{t}(l)$ still decays exponentially, $P_{l}(l)$ does not, but has another peak at $l_{s} \approx 200$. This peak is not a finite size effect (which can be shown using a larger system) and indicates the presence of an internal spatial structure with characteristic length $l_{s}$ (typical separation between the "nuclei") in the STI state. The typical width of the "nuclei" is, in turn, determined by $P_{t}(l)$.

Numerical data for a larger system $(L=4096)$ suggest (in contrast with the results obtained by Chate and Manneville ${ }^{19}$ ) a crossover type of behavior for the distribution of laminar domain lengths away from the onset of STI:

$$
P_{l}(l) \sim\left\{\begin{array}{l}
l^{\alpha}, \alpha<0, \quad \text { for } 1 \ll l \ll l_{s}, \\
\exp \left(-l / l_{s}\right), \text { for } l \gg l_{s} .
\end{array}\right.
$$

As a result, if the characteristic length $l_{s} \rightarrow \infty$ as $u \rightarrow u_{n}^{+}$we should expect a pure power law decay of $P_{l}(l)$ at $u=u_{n}$.

Another interesting phenomenon can be pointed out in fig. 4(c). Both $P_{l}(l)$ and $P_{t}(l)$ have two branches, one corresponding to even, the other to odd length of a domain. The behavior of these branches is quite peculiar, they cross at some crossover length $l_{c r} \approx 36$ : 

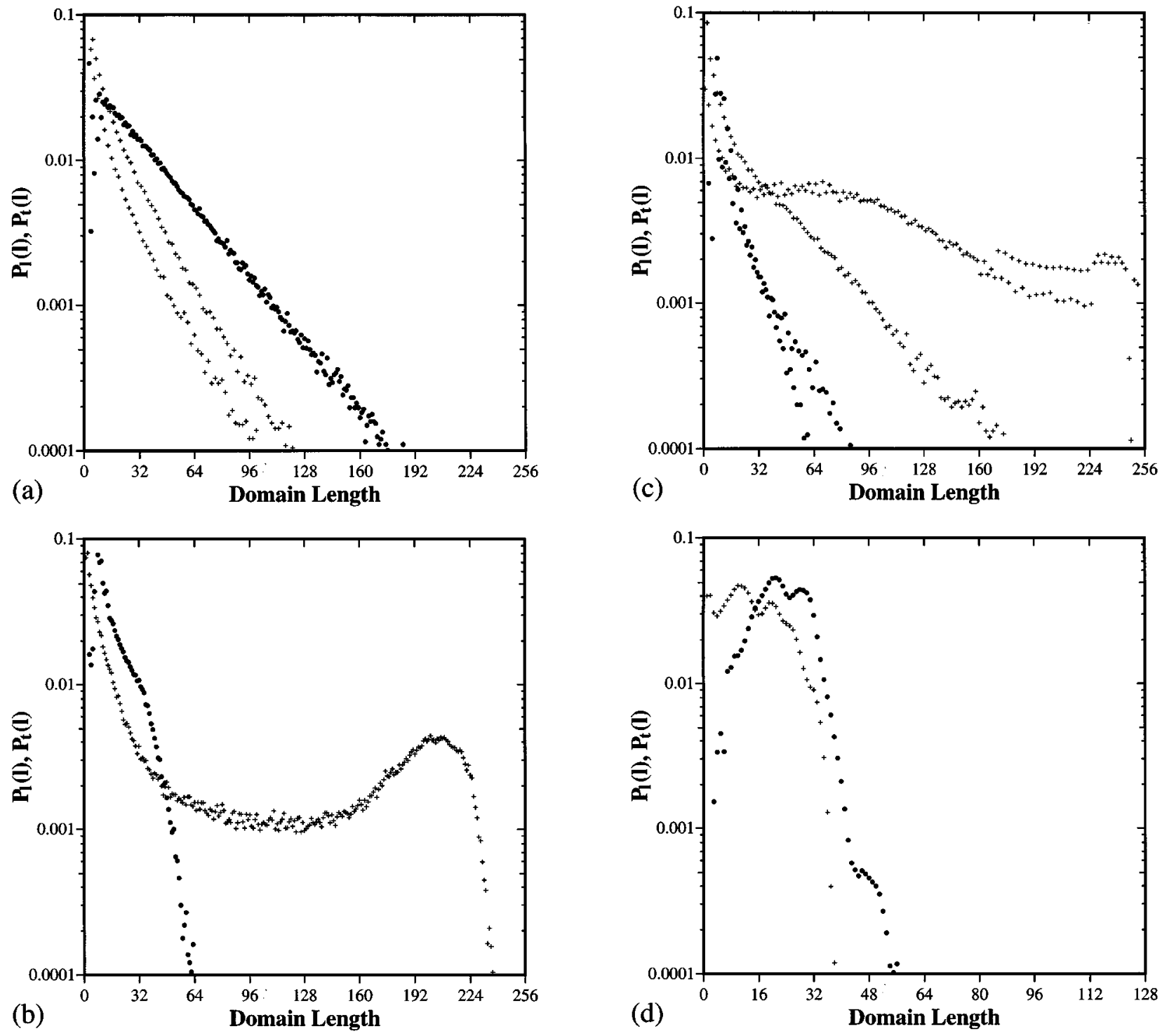

FIG. 4. Domain length probability distribution $\left(+-\right.$ laminar $P_{l}(l)$, $u=0.09$, (c) defect dominated state at $u=0.46$, (d) frozen pattern at $u=0.66$. Lattice size is 256 .

$$
\begin{aligned}
& P_{l}^{\text {even }}(l)>P_{l}^{\text {odd }}(l), \quad l<l_{c r} ; \\
& P_{l}^{\text {even }}(l)<P_{l}^{\text {odd }}(l), \quad l>l_{c r} .
\end{aligned}
$$

The difference between the branches is larger for $L$-even and smaller for $L$-odd. Similar relations hold for $P_{t}(l)$, though the difference between the branches is less pronounced than in the case of $P_{l}$.

Close to the boundary T2-L1 a typical state [fig. 3(c)] is composed of a collection of laminar domains separated by small turbulent defects. As we are going to see later, most of the turbulent domains tend to have a fixed length $l_{t}=6$, independent of the distance (in parameter space) to the transition point.

All defects move with a constant velocity, but while the majority of defects is moving with the maximal speed $v= \pm 1$ the rest have a smaller speed $|v| \leqslant 1$. Therefore we have a considerable probability of encountering a laminar domain bounded by a pair of defects which move with equal and opposite speeds. If at some particular time $n$ the length of such a laminar domain was even (odd), it will remain even (odd) for as long as these defects exist since the length will increase (decrease) by 2 at each time step.

It is reasonable to assume that the details of the "defect interaction" favor the creation of domains with length of a given parity, say even over odd. As a result, one will see that the probability of finding a laminar domain of small and even size $l$ is higher than the probability of finding a laminar domain with comparable odd size $l-1$ (or $l+1)$, thus splitting the function $P_{l}(l)$ into two branches.

Since chaotic fluctuations tend to destroy the deterministic predictions like the one we just discussed, we cannot make any rigorous conclusions about the dynamics of larger laminar domains. The numerical data suggests that the char- 
acteristic lengthscales determining the decay rates of the two branches are different, so they cross at some crossover length $l_{c r}$ determined by the average distances between the defects moving with different speeds.

In the case of a frozen pattern one should not expect the probability density to decay exponentially, rather it should display several peaks, broadened due to the chaotic fluctuations of the domain boundaries, at the lengths occurring in a particular pattern and then go sharply to zero. This applies to both $P_{t}$ and $P_{l}$. Figure 4(d) satisfies this prediction quite well. The underlying reason of this kind of behavior is the non-ergodicity of the system in the frozen pattern forming regime.

The numerical results in fig. 4 imply that in the model studied in this paper simply the ergodicity of the system dynamics is a sufficient condition for the probability distribution function $P_{t}(l)$ to decay exponentially at lengths greater than the correlation length $\xi$ in any disordered state. On the contrary, the distribution of laminar domains is more specific and informative. $P_{l}(l)$ still decays exponentially in the strongly chaotic states. In the spatiotemporally intermittent state however the behavior of $P_{l}$ varies widely: it might or might not decay exponentially. For instance, in case of the "nuclear" STI state we observe the crossover from exponential to power law type of decay. Similarly to Ref. 19 we expect to see a pure power law decay at the transition point $u=u_{n}$.

\section{PHASE TRANSITIONS}

\section{A. Order parameters}

As mentioned above, we expect to have 4 distinct phase transitions, in which the system goes from either uniform state or 2-cycle to a chaotic state. The transition to chaos in this example of an extended system with a local conservation law does not follow the period doubling cascade or other routes to chaos characteristic of low dimensional dynamical systems, instead the system goes directly from a simple dynamical state (fixed point, period-2) to a chaotic state. In other words it has a character similar to phase transitions in Hamiltonian statistical systems where a symmetry of a basic state is destroyed upon crossing of the critical point. This feature is common to all the phase transitions in this model.

A conventional dynamical systems approach to the treatment of phase transitions in a deterministic chaotic system would be to calculate the maximal Lyapunov exponent, $\lambda_{\max }$. The bifurcation from the ordered to disordered state then occurs at the values of the parameters where the exponent changes sign and becomes positive. In the case of CML with a conservation law, one of the exponents is always zero, therefore $\lambda_{\max }$ does not change sign, but increases (continuously or discontinuously, depending on the type of transition) from zero as the system crosses the boundary between the ordered and the disordered phase. The maximal Lyapunov exponent can be considered as an example of a global (intensive) order parameter.

On the other hand, in order to get some additional insight into the spatial dynamics of the system, it might be advantageous to introduce a local (extensive) order parameter. A good candidate seems to be the density $h$ of the Kolmogorov-Sinai entropy, $S_{K S}$ :

$$
h=\frac{1}{L} S_{K S}=\frac{1}{L_{\lambda_{m}}>0} \sum_{m} .
$$

It is clearly zero in the ordered phase and positive in the disordered phase.

The calculation of the Lyapunov spectra is numerically costly, so a different approach is used for comparison, based on the reduced description of the system's dynamics. In terms of reduced dynamics the phase transition from the ordered to the disordered state can be represented as the appearance of a set of disjoint turbulent domains on the laminar background. This leads us naturally to the measure of the set of turbulent domains, $\rho_{t}$, as another local order parameter, describing how the laminar state becomes turbulent. In the ordered state $\rho_{t}=0$, in the disordered state $0<\rho_{t}<1$.

And, finally, let us introduce yet another order parameter, $e_{c h}$. One can decompose the chaotic dynamics into modes using the Karhunen-Loeve decomposition. ${ }^{22}$ The mode intensity $E_{k}$ is defined as the eigenvalue of the integral equation,

$$
\sum_{i=1}^{L} K(j, i) \psi_{k}(i)=E_{k} \psi_{k}(j),
$$

where the kernel

$$
K(i, j)=\left\langle u_{i}^{n} u_{j}^{n}\right\rangle_{n}
$$

is just the 2-point correlation function. Due to the translational invariance of the system (in case of ergodic dynamics) $K(i, j)=C(i-j)$ and therefore the eigenfunctions $\psi_{k}(i)$ are just Fourier modes. Consequently, the eigenvalues $E_{k}$ are given by the values of the static structure function,

$$
E_{k}=S(k)=\left\langle\left|u_{k}^{n}\right|^{2}\right\rangle_{n},
$$

where $u_{k}^{n}$ is the Fourier transform of the map variable.

The total intensity of the dynamics is defined as $E=\Sigma_{m} E_{m}$. It includes the contributions from the chaotic as well the non-chaotic modes.

In the non-chaotic phases L1 and L2 the stable stationary state is given by the general formula (5). Therefore, the structure function might only be non-zero at two values of the wave vector $k$ :

$$
S(0)=u^{2},
$$

and, for a zig-zag state,

$$
S(\pi)=A^{2} .
$$

In other words, if we want the order parameter to represent the strength of chaos in the system, it should be defined through the intensity of the chaotic modes only. So we arrive at the following expression:

$$
e_{c h}=\frac{1}{L} \sum_{m=1}^{L / 2-1} S\left(k_{m}\right), \quad k_{m}=\frac{2 \pi m}{L},
$$




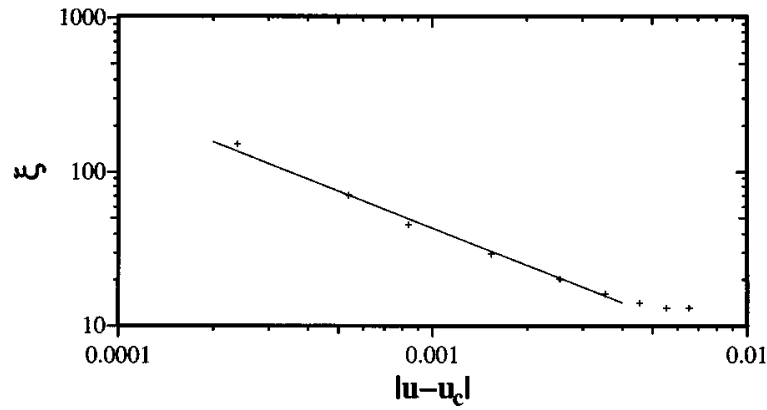

FIG. 5. Correlation length diverges near the continuous phase transition. The data for the lattice with 2048 sites is used.

which is identically equal to zero in any ordered phase and larger than zero in any disordered phase.

All order parameters introduced are expected to become asymptotically independent of the system size $L$ in the thermodynamic limit $L \rightarrow \infty$.

\section{B. Continuous transition}

Most of the attention in this study was devoted to the order-disorder transition occurring at the boundary L1-T2. This transition is easy to study and it is expected to be quite common in models described by a CML with diffusive coupling and conserved map variable density.

The transition point is defined by the equation $f^{\prime}\left(u_{c}\right)=1 / 2$. One can see from eq. (6) that at $u=u_{c}$ a period 2 bifurcation occurs and the $k=\pi$ mode becomes growing, making the uniform configuration unstable.

Details of the transition (see the next section) suggest that this transition is a continuous (second order) phase transition, and the numerical data support this conclusion. One of the clear indications of this fact is presented in fig. 5, which shows that the correlation length diverges as we approach the phase transition point,

$$
\xi \propto\left(u_{c}-u\right)^{-\nu},
$$

with critical exponent $\nu$ estimated to be of order 0.8 . The correlation length is hard to measure however, and the precision of this result is low, so that this value cannot be considered reliable.

A better diagnostic of a diverging lengthscale is the average length of a laminar domain $l_{l}=\Sigma_{l} l P_{l}(l)$ which is seen to scale $[$ fig. $6(\mathrm{a})]$ as

$$
l_{l} \propto\left(u_{c}-u\right)^{-\mu}, \quad \mu \approx 1.0 \pm 0.02,
$$

which implies, in particular, that very close to the transition point the lattice configuration consists of a few large laminar domains, separated by turbulent defects of finite size.

The average length of a turbulent domain $l_{t}=\Sigma_{l} l P_{t}(l)$ does not scale [fig. 6(b)], but shows an exponential dependence on the distance from the critical point:

$$
l_{t} \approx l_{t}^{c r} \exp \left(\frac{u_{c}-u}{u_{t}}\right), \quad u_{t} \approx 0.006,
$$

approaching the limiting value

$$
\lim _{u \rightarrow u_{c}} l_{t}=l_{t}^{c r}=6 .
$$

This means that the onset of disorder at this particular phase transition is dominated by the creation of defects of the fixed width $l_{t}^{c r}$. In particular, one can check that the width of all the defects in fig. 3(c) is about 6 lattice spacings.

All of the order parameters defined above take a zero value in the non-chaotic phase and increase continuously from zero in the chaotic phase as we move away from the transition point. What is more interesting, they scale algebraically with the deviation of $u$ from the transition point (fig. 7), all with different critical exponents:

$$
\begin{aligned}
& \lambda_{\max } \propto\left(u_{c}-u\right)^{\beta_{\lambda}}, \quad \beta_{\lambda} \approx 0.8 \pm 0.03 ; \\
& h \propto\left(u_{c}-u\right)^{\beta_{h}}, \quad \beta_{h} \approx 2.0 \pm 0.05 ; \\
& \rho_{t} \propto\left(u_{c}-u\right)^{\beta_{\rho}}, \quad \beta_{\rho} \approx 1.0 \pm 0.01 ; \\
& e_{c h} \propto\left(u_{c}-u\right)^{\beta_{e}}, \quad \beta_{e} \approx 0.5 \pm 0.01 .
\end{aligned}
$$

One can use these values to compare the transition with similar phase transitions in other conserving systems, both continuous and discrete, and perhaps determine whether this transition belongs to some universality class.

\section{Critical exponents}

A closer look at the details of the phase transition at $u=u_{c}$ reveals that equation (35) is to be expected and that the transition should necessarily be continuous, as a result of the conservation law and a particular feature of the local map $f(x)$.

As was mentioned in the previous section, the change in the growth rate of the $k=\pi$ mode is responsible for the transition. One can determine from eq. (6) that for $u$ close to $u_{c}$, equating the Lyapunov exponent with the growth rate, the linear stability analysis of the uniform state gives

$$
\lambda_{\pi} \approx-8 b\left(u-u_{c}\right) \text {, }
$$

which changes sign as the system moves across the transition point, from the ordered state L1 to disordered state T2. At the transition point the growth rate obviously vanishes, making the zig-zag state neither stable nor unstable in the linear sense. In fact as mentioned in section II, the zig-zag state given by eq. (5) (type-II 2-cycle) with arbitrary amplitude $A$ can exist at $u=u_{c}$ (and only at $u=u_{c}$ ) and is stationary, meaning that the amplitude $A$ neither grows nor decays. This is in contrast with the result for phase L2 (type-I 2-cycle), where the amplitude of the stable state is defined by eq. (10).

This fact results in some interesting consequences for the system dynamics in the disordered phase close to the transition point. Most of the lattice develops a zig-zag pattern (fig. 8) similar to the one we just discussed,

$$
u_{i}^{n}=u_{c}+(-1)^{i+n} A_{i},
$$

where now the amplitude $A_{i}$ is not a constant, but a slowly varying function of the lattice site. The whole lattice cannot be in such a state for $u<u_{c}$ because of the conservation law. In order to compensate for the difference, several similar 

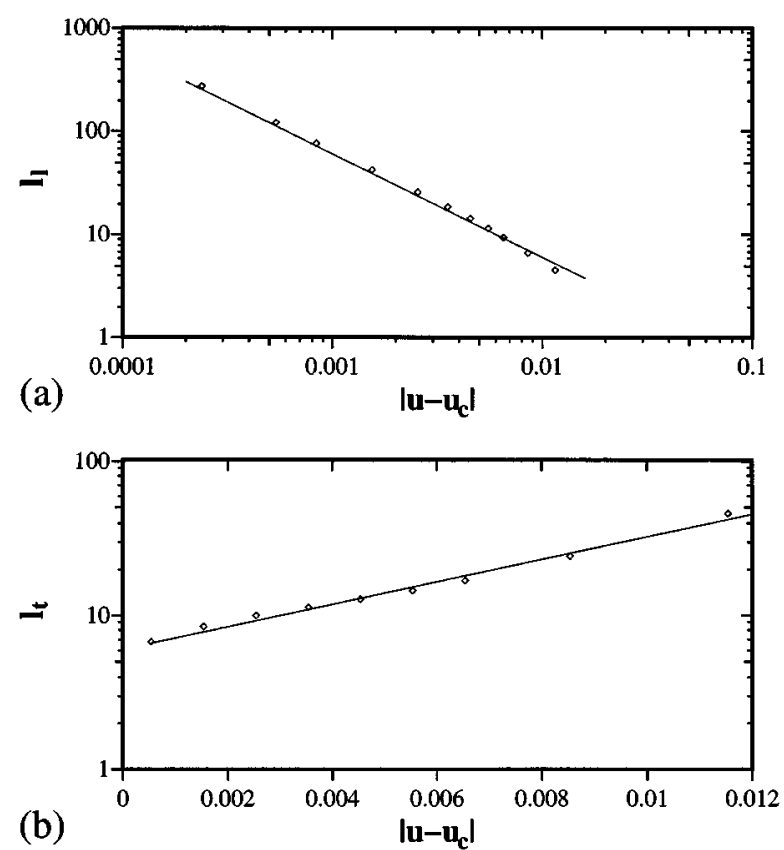

FIG. 6. Critical behavior of average domain lengths: (a) length of laminar domains diverges algebraically, (b) length of turbulent domains converges exponentially.

turbulent defects separating laminar domains form, with a fixed width $l=l_{t}^{c r}$ (see the previous section) and the local density of the map variable,

$$
\frac{1}{l} \sum_{i=i_{0}}^{i_{0}+l-1} u_{i}^{n}=u_{c}-\delta u
$$

which is lower than the critical value $u_{c}$ by $\delta u$.
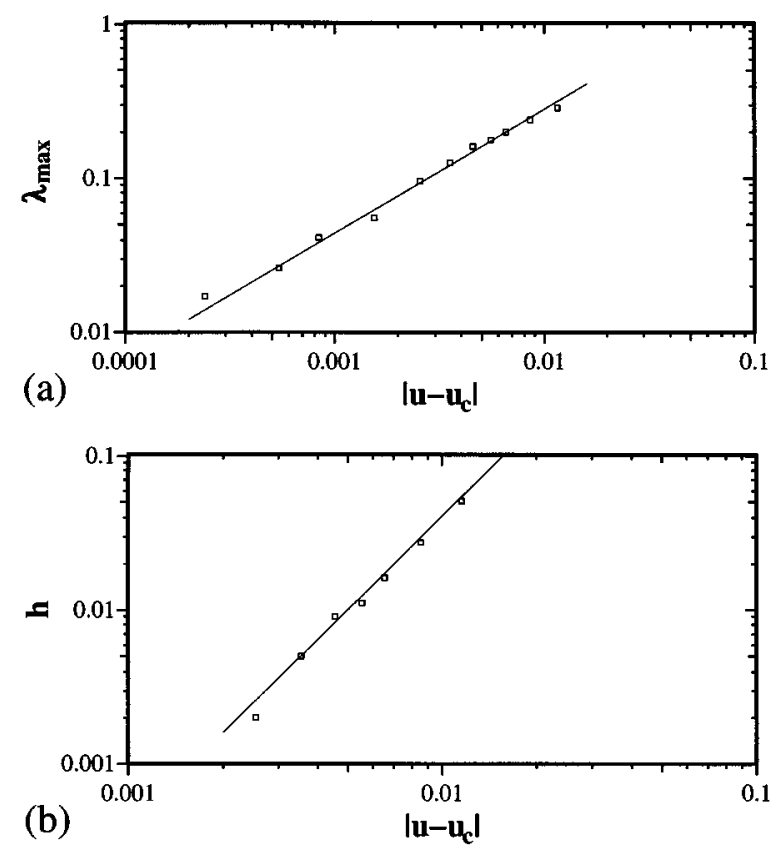

Here $\delta u$, should not strongly depend on $u$, because $u$ is not a local parameter prescribing the dynamics. On the contrary, the local density in the turbulent defect is only determined by the structure of the interface separating two laminar domains that have their local densities fixed at $u_{c}$, independent of $u$. The structure of the interface, in turn, depends primarily on the width of the turbulent domain, which is seen to depend very weakly on $u$. Numerical results support this conclusion.

This results in the value of the conserved quantity being "adjusted" to comply with the conservation law to give on average

$$
\rho_{l} u_{c}+\rho_{t}\left(u_{c}-\delta u\right)=u .
$$

Now we can easily extract the dependence of $\rho_{t}$ on $u$. Since $\rho_{t}+\rho_{l}=1$,

$$
\rho_{t}=\frac{\left(u_{c}-u\right)}{\delta u} .
$$

This derivation confirms the value of the critical exponent $\beta=1$. Thus the conservation law is ultimately responsible for the way this particular phase transition occurs and for its type. The ordered state turns into a disordered one by developing a set of very similar turbulent domains (defects), which have a fixed length [the deviation in eq. (32) from the $l_{t}=6$ is due primarily to "defect interaction" effects], but whose number increases as the system moves further and further away from the transition point, so as to compensate for the change in the density of the map variable in the laminar regions with respect to the average value given by the conservation law. We may therefore suggest that defects play (c)

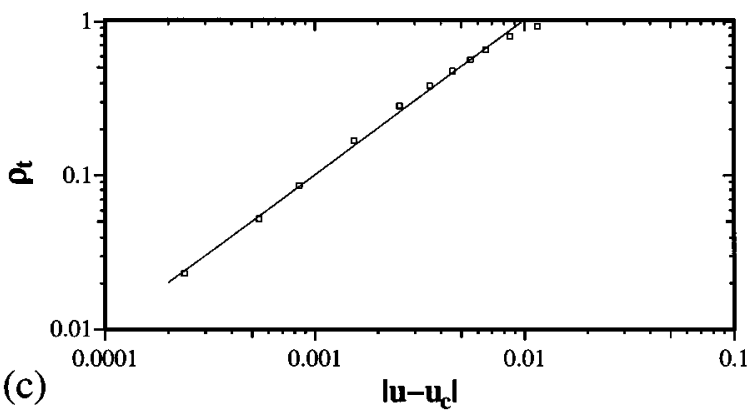

(d)

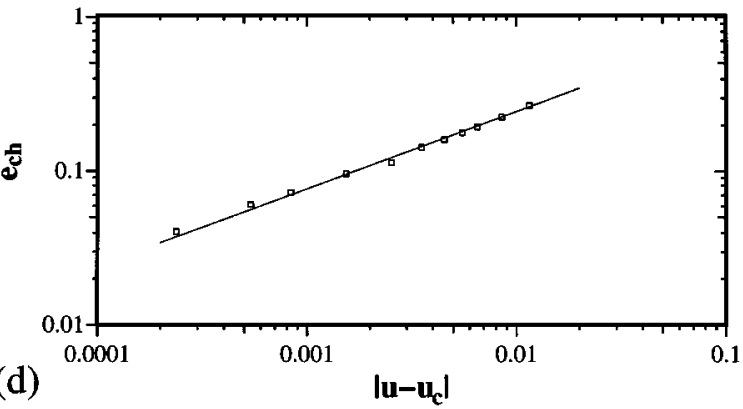

FIG. 7. Scaling of order parameters at the continuous phase transition calculated on the lattice with 2048 sites: (a) maximal Lyapunov exponent $\lambda_{\text {max }}$, (b) Kolmogorov-Sinai entropy density $h$, (c) measure of the turbulent set $\rho_{t}$, (d) intensity of chaotic modes $e_{c h}$. 
a more important role in order-disorder transitions in conserving systems than in non-conserving systems. ${ }^{17}$

The value of the critical exponent for the maximal Lyapunov exponent $\beta_{\lambda} \approx 0.8$ numerically obtained in eq. (33) is different from the one predicted by eq. (37). However the latter value is calculated for a reference trajectory corresponding to the uniform configuration and since for $u<u_{c}$ the system is in the chaotic state, the validity of eq. (37) is far from being obvious, no matter how small the distance to the transition point is. We should also consider finite size effects. It is natural to expect that numerical and theoretical values agree if there is just one positive Lyapunov exponent and therefore no mode mixing. From eq. (6) it follows that the next mode to become growing is the mode with $k=\pi-(2 \pi / L)$ and this happens when

$$
u=u_{c}-\Delta u, \quad \Delta u \approx \frac{\pi^{2}}{16 b L^{2}} .
$$

So we might expect the crossover behavior for the exponent $\beta_{\lambda}$ :

$$
\begin{aligned}
& \beta_{\lambda} \approx 0.8, \quad u_{c}-u>\Delta u, \\
& \beta_{\lambda}=1.0, \quad u_{c}-u<\Delta u .
\end{aligned}
$$

In practice the value of $\Delta u$ was usually so small ( $\Delta u \approx 3 \times 10^{-5}$ for $L=128$ ), that eq. (43) was satisfied for all deviations of $u$ from the critical value used in our numerical calculations (at most $2 \times 10^{-4}$ ).

And finally we would like to mention that the value of the critical exponent $\beta_{h}$ can be evaluated from the limiting form of the Lyapunov spectrum. Close to the transition point the positive Lyapunov exponents are well approximated by the following expression:

$$
\lambda_{m}=\lambda_{\max }-c_{1} m^{\gamma}, \quad \gamma=0.7 \pm 0.1 .
$$

Together with the equations (22) and (33) this implies that the critical exponent corresponding to the KolmogorovSinai entropy density is given by

$$
\beta_{h}=\beta_{\lambda}\left(1+\frac{1}{\gamma}\right),
$$

that yields the value $\beta_{h}=1.94 \pm 0.20$ consistent with eq. (34).

We might expect these exponents to define a universality class for the onset of spatiotemporal chaos. The arguments leading to the predictions for the values suggest that the class may depend both on the existence of a conservation law and on special symmetry properties of the map function. The restrictions on $f(x)$ can be obtained in the following way.

One starts with the relation between the amplitude and the local density of the type-II 2-cycle for an arbitrary $f(x)$ :

$$
\sum_{n-o d d} \frac{1}{n !} A^{n-1} f^{(n)}(u)=\frac{1}{2} .
$$

Close to the transition point $u=u_{c}$ it can be rewritten as

$$
0=-r\left(u-u_{c}\right) A+d_{3} A^{3}+d_{5} A^{5}+\cdots,
$$

where $r=f^{\prime \prime}\left(u_{c}\right)$ and coefficients $d_{n}$ are defined as

$$
d_{n}=\frac{1}{n !} f^{(n)}\left(u_{c}\right), \quad n \geqslant 3
$$

Depending on the values of parameters $r$ and $d_{3}$, we can have either a subcritical or supercritical bifurcation at the transition point. The special case studied in this paper corresponds to

$$
d_{3}=d_{5}=\cdots=0,
$$

and is intermediate between the two types of bifurcation.

These special properties follow immediately for continua of $a$ and $b$ from the parabolic nature of the map we have used. More generally we can consider maps of the form

$$
f(x)=a x+b s(x),
$$

where $s(x)$ is an arbitrary function symmetric about its maximum. By rescaling and shifting the origin of $x$ and choice of normalization of $s$ we can set $s(0)=0$, the maximum of $s$ to occur at $s=1 / 2$ and then $s(1 / 2)=1$, leaving the two parameters $a$ and $b$ as well as the conserved quantity $u$ to define the system. For this general family of maps the degenerate bifurcation to the period- 2 state occurs only for $a=1 / 2$ and for $u$ at the maximum of $s$, i.e. $u=1 / 2$. Thus the universality class is codimension-2 - two parameters must be tuned to arrive at this type of transition.

For other values of $a$ and $b$ in (51) the bifurcation to the period-2 state will be either supercritical or subcritical. For the supercritical case a stable laminar 2-cycle state develops, which may be the first step in a subharmonic cascade. For the subcritical case attractors develop far away in phase space, and a full non-linear analysis is needed to determine the type of behavior.

\section{Hysteretic transitions}

Now we turn our attention to the phase transitions that we expect to occur at the boundaries L2-T1, L2-T2 and L1-T1. As we are going to see later, all three are very similar, so we will concentrate on the transition at L2-T1 below.

There is a considerable difference between the transition at L1-T2 and the transition at L2-T1: in the former case the asymptotic state in both L1 and T2 is unique, while in the latter case the asymptotic state in the ordered phase L2 can be either ordered or spatiotemporally chaotic. As a result we should specify between which states the transition occurs.

It will be convenient to introduce an additional parameter $v(u)$ characterizing the volume of the of the basin of attraction. For example, as we know from section IV in the sub-phase $L 2_{l}\left(u_{p}<u<u_{n}\right)$ the attractor is unique and therefore the basin of attraction is the whole configuration space with volume $v_{l}(u)=1^{L-1}=1$.

In the sub-phase $L 2_{p}$ the non-chaotic attractor coexists with the chaotic one, so we have $0<v_{l}(u)<1$ for $u_{a}<u<u_{p}$. Since there are no other attractors in this subphase, the volume of the basin of attraction of the chaotic attractor is given by $v_{t}(u)=1-v_{l}(u)$. Numerical data suggest that most of the initial conditions in $L 2_{p}$ result in spa- 
tiotemporally intermittent chaotic asymptotic state and therefore typically $v_{t}(u) \gg v_{l}(u)$. Moreover $v_{l}(u) \rightarrow 0$ as $u \rightarrow u_{a}^{+}$ while $v_{t}(u) \rightarrow 0$ as $u \rightarrow u_{p}^{-}$.

At the point $u=u_{a}$ the 2-cycle state loses its stability through a subcritical bifurcation. Outside the phase L2 the 2-cycle state cannot exist and as the system crosses the phase boundary all order parameters jump from zero in the phase L2 to some non-zero values in the phase T1 (see fig. 2). As a result one observes a discontinuous transition in the nonchaotic state: the laminar state abruptly turns into the chaotic one.

However, there is apparently no phase transition in the chaotic state at this point: the dynamics of the system on the $\mathrm{T} 1$ side of the boundary is very similar to the dynamics of the system evolving on the chaotic attractor on the L2 side. All order parameters change continuously as the system crosses the boundary from L2 to T1. Since $v_{l}(u) \rightarrow 0$ as $u \rightarrow u_{a}^{+}$, changing the direction does not modify this conclusion: the chaotic attractor of the phase T1 smoothly transforms into the chaotic attractor of the phase L2.

At $u=u_{p}$ the situation is reversed. Obviously there could be no phase transition in the non-chaotic state. On the other hand, the chaotic attractor does not exist in $L 2_{l}$, therefore there should be some kind of phase transition in the chaotic state: the order parameters are zero for $u_{p}<u<u_{n}$, but take on non-zero values in the chaotic state for $u_{a}<u<u_{p}$. Thus $u=u_{p}$ corresponds to the onset of STI.

The coexisting attractors form a hysteresis loop in the sub-phase $L 2_{p}$ (fig. 2). If we start at $u>u_{p}$ and gradually decrease parameter $u$ the system will remain in the nonchaotic state while $u>u_{a}$ and then jump to the chaotic one at $u=u_{a}$. Conversely starting at $u<u_{a}$ and gradually increasing parameter $u$ makes the system remain in the chaotic state while $u<u_{p}$. At $u=u_{p}$ the chaotic state becomes nonchaotic thus closing the loop.

There is a numerical complication here: it is not possible to establish the exact value of the critical parameter $u_{p}$ for a system of finite size. For $u \rightarrow u_{p}^{+}$the lifetime of the chaotic transients becomes very long and one cannot reliably determine the type of the asymptotic state. On the other hand, $v_{t}(u) \rightarrow 0$ as $u \rightarrow u_{p}^{-}$, which means that it becomes increasingly hard to find initial conditions resulting in a persistent chaotic state as $u$ gets close to $u_{p}$, especially for small systems. Although $v_{t}(u)$ grows rapidly with the system size, the smaller $\left|u-u_{p}\right|$ is the larger the system should be in order to obtain the persistent chaotic state. Therefore one cannot reliably determine the values of the order parameters in the chaotic state close to $u=u_{p}$.

As a result, it is even hard to determine reliably whether the transition at $u=u_{p}$ in the chaotic state is actually continuous, although the correlation length seems to diverge approaching the transition point. The order parameters do not provide a clear picture either. Both $h$ and $\rho_{t}$ seem to increase continuously from zero, but $\lambda_{\max }$ and $e_{c h}$ jump discontinuously as the system moves across the transition point to the chaotic sub-phase $L 2_{p}$. All order parameters gradually increase as the system moves away from the transition point toward the chaotic phase T1. The number of positive
Lyapunov exponents also grows showing the increase in the number of chaotic modes. These results are consistent with Ref. 24. But so far we do not have reliable data that will allow us to determine whether this transition in fact belongs to the universality class of directed percolation or not.

There is a similarity between the transition at $u=u_{p}$ and the continuous phase transition at $u=u_{c}$ : the laminar state becomes chaotic through the appearance of a set of turbulent domains that gradually spread over the whole system. Nevertheless there is an important difference: the STI state observed inside T2 can never become completely laminar because of the conservation law, while the STI state inside $L 2_{p}$ may decay into a completely laminar state with time.

The above discussion applies completely to the chaotic sub-phase $L 2_{n}$ which corresponds to $u_{n}<u<u_{b}$. One just has to replace T1 with T2, $u_{p}$ with $u_{n}$ and $u_{a}$ with $u_{b}$. In particular, $v_{l}(u) \rightarrow 0$ as $u \rightarrow u_{b}$. One particular feature of the chaotic attractor in this sub-phase is worth mentioning: for $0.1 \lesssim u \lesssim 0.15$ the measure of the turbulent set, $\rho_{t}$, grows linearly with $u$.

The phase transition L1-T1 at $u=u_{d}$ is also very similar to the transition L2-T1. One just has to replace $L 2_{p}$ with $L 1_{f}, u_{p}$ with $u_{f}$, and $u_{a}$ with $u_{d}$ in the above discussion.

Here, unlike the phase L2, the laminar state is the uniform state, not the 2-cycle. The uniform state is unstable for $u>u_{d}$, so in order for the laminar regions to be stable the local density inside them should be in the range $u_{c}<u<u_{d}$ characteristic of the ordered phase L1. This is in fact the case: the value of the local density in the laminar regions is $u_{l} \approx 0.53$.

Inside $L 1_{f}$ the number of positive Lyapunov exponents grows linearly with $\rho_{t}$, while the latter grows linearly with $u$. This is the kind of behavior one expects in a system where all chaos is localized in turbulent domains.

All three cases of hysteretic transitions observed in our model are very similar and represent many of the characteristic features of the specific route to chaos. In particular, STI appears whenever the uniform absorbing state experiences a subcritical bifurcation at the transition point (e.g., $u=u_{a}$ ); the hysteresis loop forms as a result of the bistability; onset of STI (e.g., at $u=u_{p}$ ) can be either continuous or discontinuous, depending on the types of defects supported by the laminar (absorbing) state; the lifetime of the "laminar" transients diverges at the onset; the confinement effects enhance the stability of the absorbing state in relatively small systems.

\section{LYAPUNOV DIMENSION}

In this section we will briefly comment on the applicability of the Lyapunov dimension as a parameter characterizing the dynamics in the system under consideration. This has been used by a number of authors (e.g., Refs. 23 and 10) to assess the strength of chaos in non-linear dynamical systems.

The Lyapunov dimension is defined as

$$
D_{L}=n+\frac{\nu_{n}}{\nu_{n}+\nu_{n+1}},
$$




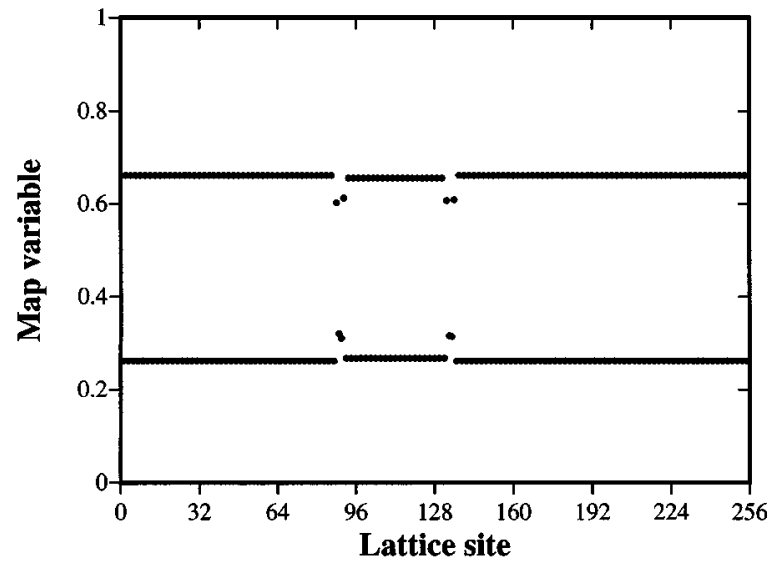

FIG. 8. Typical lattice configuration near the continuous phase transition $(u=0.46)$ : two large laminar domains with different amplitudes $A_{1}$ and $A_{2}$ are separated by two turbulent defects of the same width. Lattice size is 256.

where $\nu_{n}=\sum_{i=1}{ }^{n} \lambda_{i}$ and $n$ is such that $\nu_{n}>0, \nu_{n+1}<0$. It was suggested in Ref. 1 that $D_{L}$ is not defined for the system considered, because for the values of the control parameters used all $\nu_{n}$ 's were positive.

This conclusion appears incorrect. First of all, this only happens for some restricted set of control parameters. For other values of parameters the Lyapunov dimension is perfectly well defined. Second, there is no problem with all $\nu_{n}$ 's being positive: it only means that $D_{L}=L-1$, i.e., the dimension of the attractor coincides with the dimension of the configuration space ( $L$ variables with one constraint) meaning that the attractor fills the configuration space. As one can see from the fig. 9 this only happens away from the boundaries inside the chaotic phases $\mathrm{T} 1$ and $\mathrm{T} 2$, where the system is in the strongly chaotic regime with $\rho_{t} \approx 1$.

The numerical results show that the Lyapunov dimension thus defined is a good (albeit costly to calculate) measure of the strength of chaos in the system under consideration. It is a continuous function of control parameters and can in principle be used as an alternative order parameter, though it proved to be hard to calculate with the necessary precision close to the phase transition points.

\section{SINGULARITIES IN THE LYAPUNOV SPECTRUM}

\section{A. Introduction}

It has been suggested ${ }^{9}$ that all coupled maps with a conservation law should have a singularity in the spectrum of Lyapunov exponents at the value $\lambda=0$. The origin of the divergence of the number of Lyapunov modes with negative exponents close to zero is generally explained by the following arguments.

A spatially uniform autonomous system with a locally conserved density is considered. The sufficiently coarse grained asymptotic state of such a system is supposed to be uniform, i.e., the density in the asymptotic state should not depend on the spatial coordinates. Now a long wavelength density perturbation is imposed on such an asymptotic state.

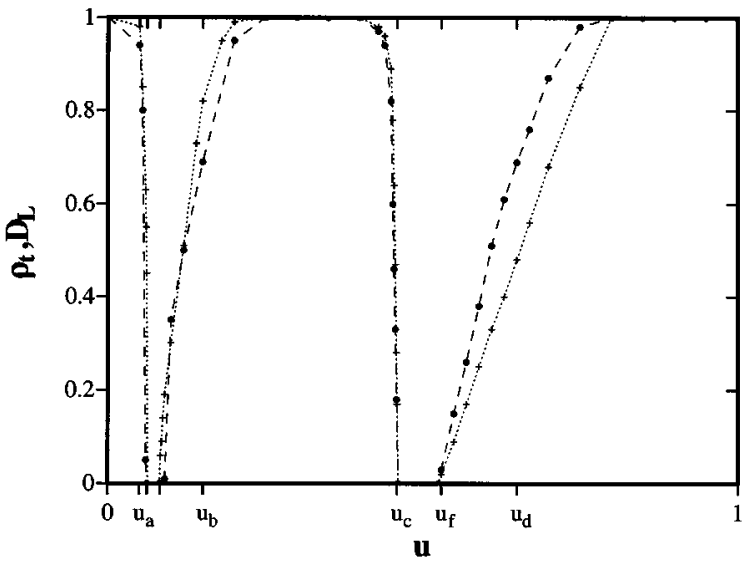

FIG. 9. Lyapunov dimension $D_{L}(\bigcirc)$ and turbulent set measure $\rho_{t}(+)$ as functions of the conserved quantity $u$, calculated on the lattices with 128 and 2048 sites, respectively. Only the values in the chaotic state are shown if two attractors coexist.

Because of the conservation law the only mechanism by which such a perturbation can decay is diffusion from the regions with high average density to the regions with low average density. This process can be described by an effective diffusion equation, which can be considered as a long wavelength approximation of the original evolution equation. As a result the decay rate of a long wavelength perturbations should be given by a quadratic function of the wave vector $k$.

In our model this functional dependency can be motivated by analogy with the analytic expression obtained for a stable non-chaotic phase L1. Since there is an exact 1-to-1 correspondence between the Fourier mode number and the decay rate in L1, we can formally rewrite eq. (6), reexpressing the number of the exponent $m$ through the wave vector $k$ :

$$
\lambda(k)=\ln \left|1-4(a+b-2 b u) \sin ^{2}\left(\frac{k}{2}\right)\right| .
$$

Here for $k \rightarrow 0$ we have $\lambda(k) \propto k^{2}$ and the density of Lyapunov exponents diverges,

$$
n(\lambda) \propto|\lambda|^{-1 / 2} \rightarrow \infty, \quad \lambda \rightarrow 0^{-} .
$$

One can attempt to apply a similar numerical analysis in the chaotic phase. We start with writing the evolution equation (1) in Fourier space:

$$
\begin{aligned}
u_{k}^{n+1}= & u_{k}^{n}-4(a+b-2 b u) \sin ^{2}\left(\frac{k}{2}\right) u_{k}^{n} \\
& +4 b \sin ^{2}\left(\frac{k}{2}\right) \sum_{k^{\prime} \neq 0, k} u_{k^{\prime}}^{n} u_{k-k^{\prime}}^{n}
\end{aligned}
$$

Consequently the Jacobian $J_{l m}^{n}=\partial u_{l}^{n+1} / \partial u_{m}^{n}$ of the evolution transformation takes on the following form in the Fourier space: 


$$
\begin{aligned}
J_{k k^{\prime}}^{n}= & \left(1-4(a+b-2 b u) \sin ^{2}\left(\frac{k}{2}\right)\right) \delta_{k k^{\prime}} \\
& +8 b u_{k-k^{\prime}}^{n} \sin ^{2}\left(\frac{k}{2}\right)\left(1-\delta_{k k^{\prime}}\right) .
\end{aligned}
$$

Fourier modes are not eigenvectors of this matrix (unless all $u_{k}^{n}=0$ as in the uniform state), but since the off-diagonal elements are of order $O\left(k^{2}\right)$ Fourier modes can serve as a good first order approximation to the exact eigenvectors for $k$ sufficiently small. This argument suggests that wave vector $k$ should be a good label for the slow modes (and small $\lambda$ ) of the system in chaotic as well as spatially ordered states.

\section{B. Structure of the Lyapunov vectors}

Numerically the Lyapunov spectrum can be calculated using the QR decomposition of the product of the Jacobians $J_{l m}^{n}$,

$$
J^{n} Q^{n-1}=Q^{n} R^{n}
$$

where $Q$ is orthogonal and $R$-upper-diagonal to yield

$$
J^{n} \cdots J^{0}=Q^{n} R^{n} \cdots R^{0}=Q^{n} \widetilde{R}^{n} .
$$

The columns of $Q^{n}$ give Lyapunov vectors and the diagonal elements of $\widetilde{R}^{n}$-the corresponding Lyapunov exponents on the $n$-th step:

$$
\lambda_{m}=\frac{1}{n} \ln \left(\widetilde{R}_{m m}^{n}\right)=\left\langle\ln \left(R_{m m}^{n}\right)\right\rangle_{n} .
$$

Figures 10-12 show several typical time averaged power spectra of the instantaneous Lyapunov vectors along with the corresponding Lyapunov spectra. The power spectra are represented in the form of the density plots showing the relative contribution $P_{m}(k)$ from the Fourier mode with number $k L / 2 \pi$ to the $m$-th Lyapunov vector, while the Lyapunov spectra show the correspondence between Lyapunov vectors and exponents. The power spectra are normalized,

$$
\sum_{k} P_{m}(k)=1
$$

so that $P_{m}(k)$ can alternatively be interpreted as the probability distribution functions.

We would like to mention that due to the ergodicity of the dynamics the power spectra (as well as Lyapunov spectra) should be reproducible, i.e., a different initial configuration with the given set of control parameters should produce the same spectrum and this is found to be the case. It is interesting to note though, that we obtained a unique form for the spectra even for the values of the control parameters producing frozen patterns, where we expect ergodicity to break down.

We start with the spectra calculated inside the chaotic phases $\mathrm{T} 1$ and $\mathrm{T} 2$. One can easily notice that a singularity appears in the spectrum of Lyapunov exponents [fig. 10(b)] when the dominant contribution to the Lyapunov vectors, corresponding to a slow evolution (small $\lambda$ ) comes from the
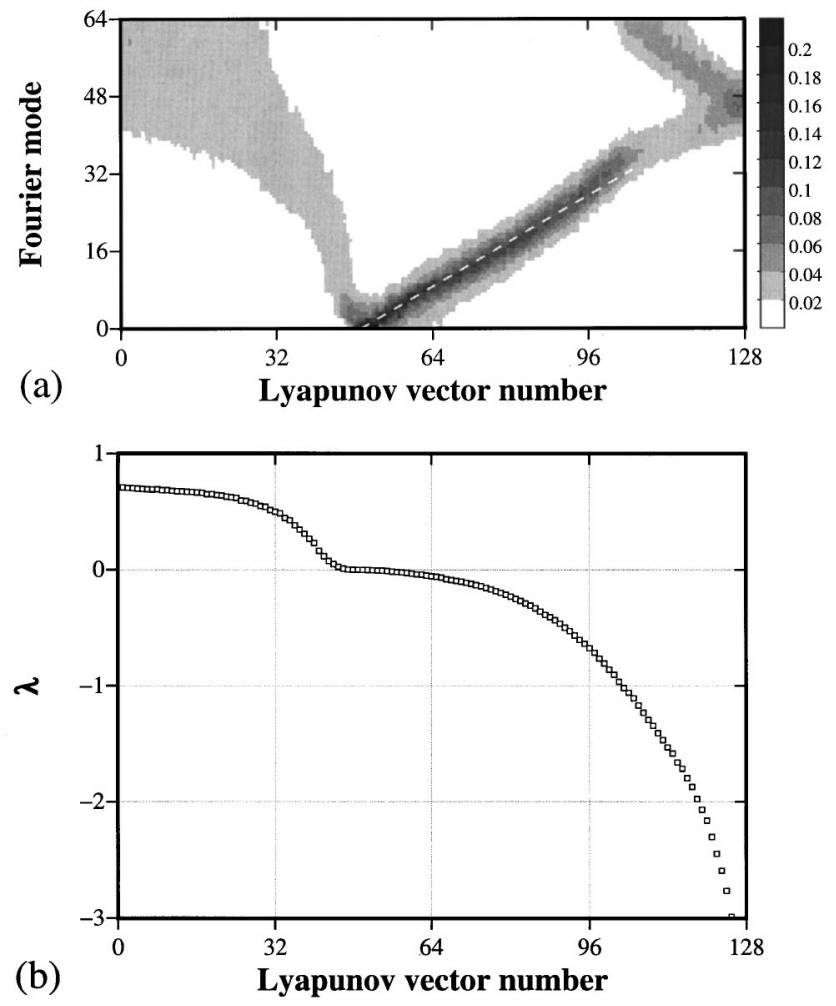

FIG. 10. Power spectrum (a) and Lyapunov spectrum (b) at $u=0.7$, inside the chaotic phase $\mathrm{T} 1$. The dominant contribution to the Lyapunov vectors corresponding to small $\lambda$ comes from the long wavelength Fourier modes only (the white dashed line gives the fit provided by eq. (63) with $\alpha=0.6$ ). As a result a pronounced singularity appears in the Lyapunov spectrum (exponent density becomes singular at $\lambda=0$ ). Lattice size is 128 .

long wavelength Fourier modes and there is at least an approximate 1-to-1 correspondence between the exponent number and the dominant Fourier mode number for the relevant vector [fig. 10(a)]. It is therefore natural to expect the small negative Lyapunov exponents to be determined by the decay rate of the corresponding Fourier mode.

In the long wavelength limit $k \rightarrow 0$, the latter could be determined from a hydrodynamic analysis ${ }^{25}$ of the problem. It was $\operatorname{argued}^{1}$ that at long wavelengths the effect of all short wavelength corrections to the equation of motion (55) can be combined into a stochastic noise term and a renormalized diffusion constant $D$, producing an effective (discrete) Langevin equation:

$$
u_{k}^{n+1}-u_{k}^{n}=-D k^{2} u_{k}^{n}+k^{2} \eta_{k}^{n},
$$

with a $\delta$-correlated noise term

$$
\left\langle\eta_{i}^{n} \eta_{i^{\prime}}^{n^{\prime}}\right\rangle=B \delta_{i i^{\prime}} \delta_{n n^{\prime}} .
$$

According to the Central Limit Theorem such noise averages to small values on large lengthscales and therefore, for small $k$, the decay rate of the Fourier mode $u_{k}$ is equal to $-D k^{2}$.

Because of the conservation law one of the exponents in our model is always equal to zero. Let it be $\lambda_{m_{0}}$. It obviously corresponds to the Lyapunov vector with $k=0$. Typically, for small $\lambda$, the relation between the number $m$ of the 


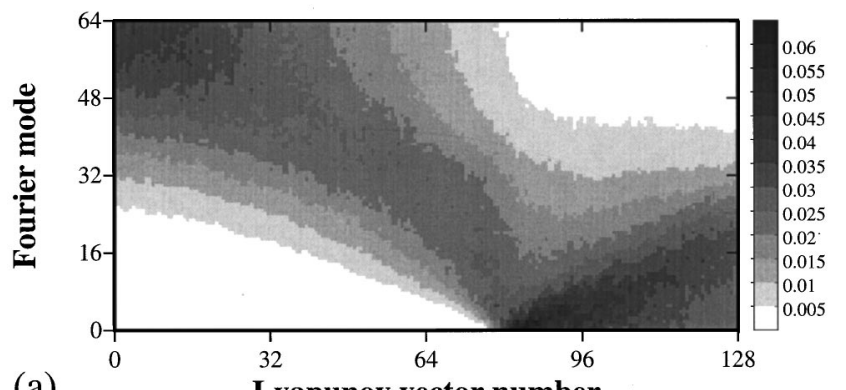

(a) Lyapunov vector number

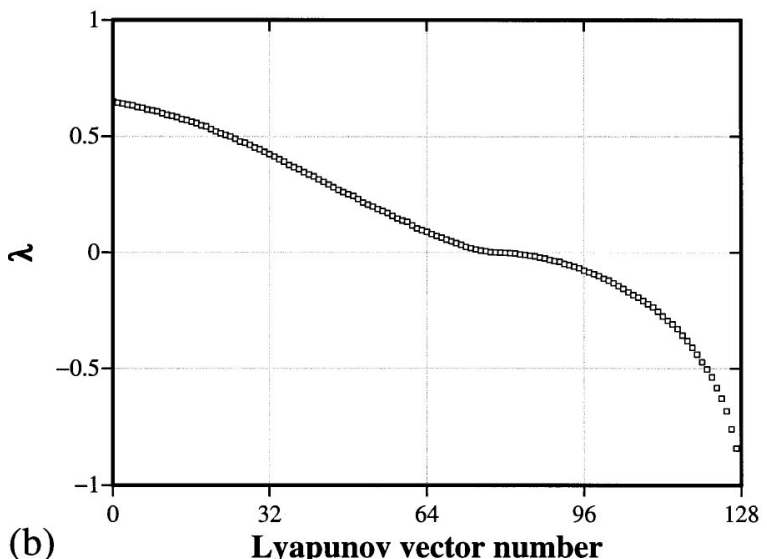

(b) Lyapunov vector number

FIG. 11. Power spectrum (a) and Lyapunov spectrum (b) at $u=0.3$, deep inside the chaotic phase T2. The dominant contribution to the Lyapunov vectors corresponding to small $\lambda$ comes from the long wavelength Fourier modes, but due to the contribution from mid-wavelength modes the singularity in the exponent density becomes much weaker. Lattice size is 128 .

Lyapunov vector and the dominant long wavelength Fourier mode $k$ can be represented by the following simple form:

$$
k_{m}=\alpha \frac{2 \pi}{L}\left(m-m_{0}\right)
$$

Parameters $m_{0}$ and $\alpha \sim 0.5$ are determined from the Lyapunov spectrum and power spectrum, respectively $\left(m_{0}=0, \alpha=0.5\right.$ inside L1).

The Lyapunov exponent $\lambda_{m}$ is in fact calculated as a time averaged decay rate of the corresponding instantaneous Lyapunov vector. Since the time averaged power spectrum $P_{m}(k)$ gives the averaged relative contribution of the Fourier mode $k$ to the $m$-th Lyapunov vector, we might estimate the value of the $m$-th exponent as

$$
\lambda_{m}=-D \sum_{k} P_{m}(k) k^{2}
$$

As one can deduce from fig. 10(a), for $u=0.7, P_{m}(k)$ is sharply peaked at $k_{m}$, so approximating $P_{m}(k)=\delta_{k_{m} k}$ we readily obtain that the form of the Lyapunov spectrum for small negative values of the exponent should be given by

$$
\lambda_{m} \approx-D k_{m}^{2}, m=m_{0}, m_{0}+1, \ldots
$$

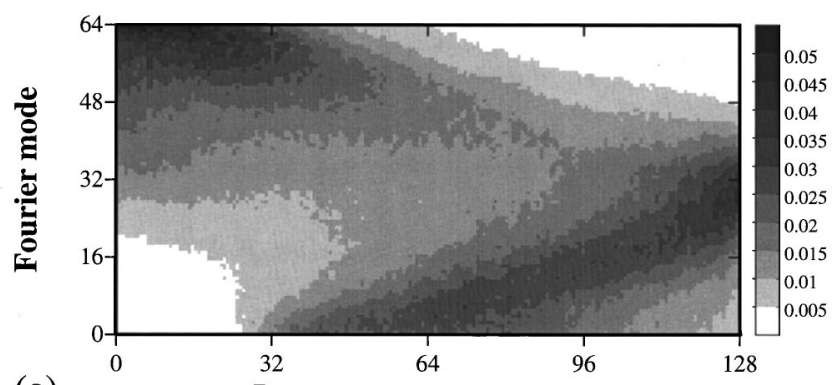

(a)

Lyapunov vector number

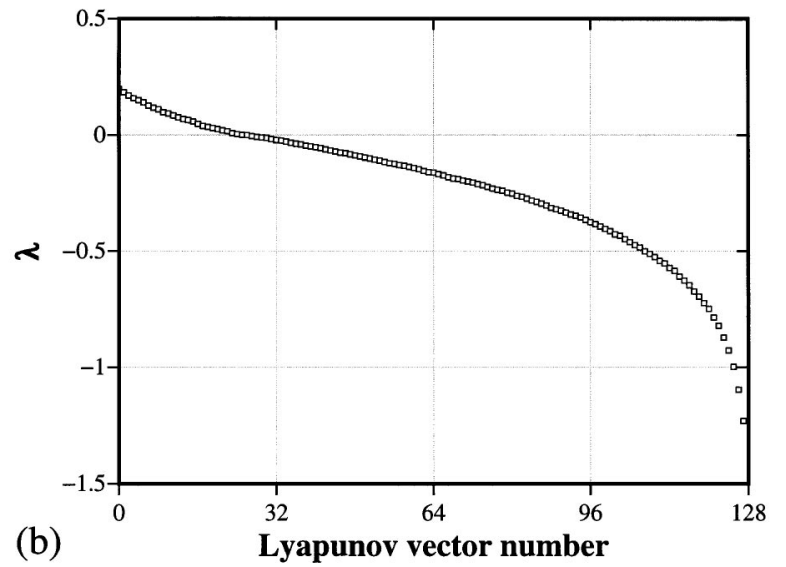

FIG. 12. Power spectrum (a) and Lyapunov spectrum (b) at $u=0.455$, close to the point where the system experiences the continuous phase transition. There is a considerable contribution from the short wavelength Fourier modes to the Lyapunov vectors corresponding to small $\lambda$ resulting in the disappearance of the singularity. Lattice size is 128 .

One can easily check that the numerically calculated exponents are in fact given quite precisely by this expression with $D \approx 0.32$ for $0<k \leq \pi / 4$. So we recover eq. (54), but now for the chaotic state.

Figure 11 provides us with another typical example of the spectra corresponding to the strongly chaotic dynamics, now calculated for $u=0.3$. One can notice that the singularity in the Lyapunov spectrum in fig. 11(b) is still present, although the quadratic fit provided by (65) is quite poor compared to the one for $u=0.7$.

The power spectrum [fig. 11(a)] shows that, similarly to the previous case, the dominant contribution to the Lyapunov vectors corresponding to slow evolution comes from the long wavelength modes, but now the distribution $P_{m}(k)$ is much broader. This means that we can no longer approximate $P_{m}(k)$ by $\delta_{k_{m} k}$ and there could be considerable corrections to eq. (65), which does not however change the general conclusion about the presence of the singularity in the spectrum of Lyapunov exponents at $\lambda \approx 0$.

These arguments work well in the area of strong chaos, where the large scale dynamics is determined by the long wavelength modes, i.e., modes with $k \ll 1 / \xi$, where $\xi$ is the correlation length in the system. When we approach a continuous phase transition, though, the correlation length grows and becomes comparable to the system size $L$. When this happens, the nonlinear terms in eq. (55) become relevant on 
every scale ${ }^{1}$ and their effect can no longer be emulated by an effective noise term. This results in a strong coupling between different long wavelength modes (i.e., modes with $k \sim 1 / L)$ and therefore the approximate 1-to-1 correspondence between the mode number and its growth rate is completely destroyed. This means that there is no reason to expect the divergence of the number of slowly evolving modes anymore.

Figure 12(a) suggests that there is a strong mode mixing in the system and it is not possible to extract the dominant contribution to the Lyapunov vectors. In fact, we really have a continuous phase transition at $u=u_{c}$, very close to $u=0.455$, and it is responsible for the disappearance of a singularity in the spectrum in fig. 12(b). One might still hope to find a trace of a singularity at this particular value of $u$ by going to much larger system size (higher resolution).

\section{Positive side singularity}

Some models with a conservation law ${ }^{9}$ are found to have a density of exponents diverging on the positive $\lambda$ side,

$$
n(\lambda) \rightarrow \infty, \quad \lambda \rightarrow 0^{+},
$$

although this feature is not considered very common. This divergence is present in our model too [see fig. 10(b) for example], but is somewhat weaker than the divergence at negative $\lambda$ : for some values of the parameters it can be barely seen even for large lattices (typically $L>10^{2}$ is necessary). For $\lambda \rightarrow 0^{+}$the spectrum can usually be fitted quite precisely by a quadratic function,

$$
\lambda_{m} \approx \widetilde{D}\left(m-m_{0}\right)^{2}, \quad m=m_{0}, m_{0}-1, \ldots,
$$

which means that the singularity of $n(\lambda)$ is inverse-squareroot on both positive and negative sides.

Numerically calculated spectra show no sign of smoothing out of the singularity (on either side) with increasing resolution (increasing size of the system) up to $L=512$ [the results for $L=128$ and $L=512$ are presented in fig. 14(a)] and also suggest that the fit (67) could be good for positive $\lambda$ as large as $0.3 \lambda_{\max }$.

Figures 10(a) and 11(a) suggest that it is possible to extract the dominant Fourier modes corresponding to small positive $\lambda$. Again their wave vectors scale roughly linearly with $m_{0}-m$ and therefore equation (63) should be replaced by

$$
k_{m}=\tilde{\alpha} \frac{2 \pi}{L}\left(m_{0}-m\right),
$$

for $m<m_{0}$. Equation (64) though should be abandoned in favor of a more precise one, preferably derived directly from the evolution equation (55). We intend to explore this question in more detail later.

\section{Effective diffusion constant}

We should also mention that the values of the effective diffusion constant $D$ numerically obtained from eq. (65) do

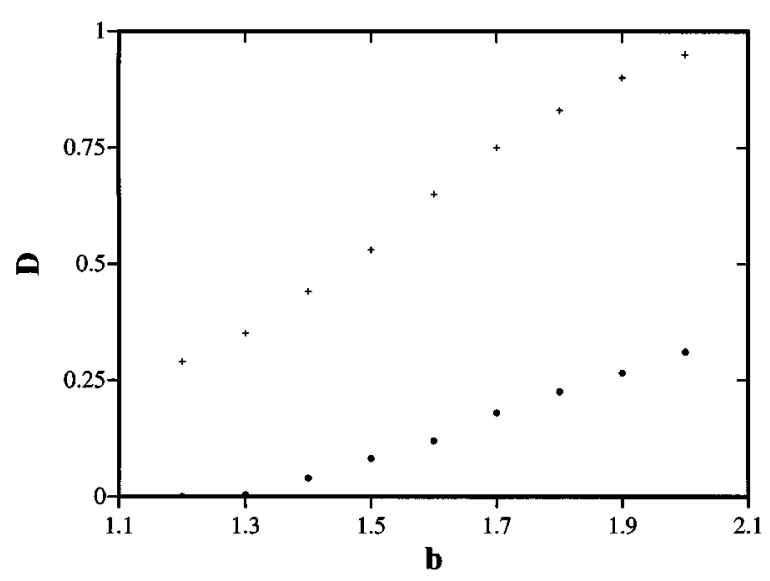

FIG. 13. Effective diffusion constant as a function of parameter $b . a=0.4$ and $u=0.8$. The values of $D_{l s}(+)$ are determined using the Lyapunov spectrum of the lattice with $L=128$, assuming $\alpha=0.5 . D_{s f}(\bigcirc)$ is obtained from the dynamic structure function calculated for $k=0.01 \pi$ on the lattice with $L=2048$.

not coincide with the ones obtained through the dynamic structure function $S(k, t)$. According to (61) it is defined (see Ref. 1) as

$$
S(k, t)=B k^{2} e^{-D k^{2} t} .
$$

It will be convenient to distinguish these using the notation $D_{l s}$ for the former and $D_{s f}$ for the latter. In the regions of parameter space where the effective Langevin equation is applicable [and hence eq. (69) is valid] these approaches can give substantially different results. This is yet another indication that equation (64) can only provide a very crude estimate and should be amended considerably to obtain adequate results.

It is instructive to compare how $D_{l s}$ and $D_{s f}$ change if parameter $b$ is varied while both $a$ and $u$ are fixed (see fig. $13)$. For $u \gtrsim 1.3 D_{l s}>D_{s f}$ and they both grow with increasing $b$. Numerical data available so far suggests that $D_{l s} / D_{s f} \rightarrow 1$ as $b \rightarrow \infty$ (for strongly chaotic systems).

For $u \leqslant 1.3 D_{s f}$ drops almost to zero indicating that there is almost no diffusion in the system. Indeed we know that this is the region of locked chaotic dynamics $\left(T 1_{l}\right)$. So this result is not surprising: the formation of a locked structure prevents diffusion (on any scale larger than some typical scale determined by that structure). We cannot probe smaller scales using the dynamic structure function because the effective Langevin equation (61) is not valid for $k$ large enough, but supposedly diffusion survives there. It is interesting to note however that locking has apparently no effect on the Lyapunov spectrum: the change in $D_{l s}$ is very gradual across $T 1$.

\section{ROLE OF THE CONSERVATION LAW}

Now that we have studied the dynamics of the CML with the conservation law quite thoroughly and compared the characteristic phenomena with those observed in CML's 
without any conservation laws we would like to discuss whether the distinguishing features are really explained by the presence of the conservation law.

It is often very hard to distinguish the effect of the conservation law on the dynamics of a system from the effects introduced by other aspects of the evolution equation. Here we would like to explore some consequences of violating the conservation law, trying to retain the structure of the original equation (1).

First of all, we are looking for internal homogeneous perturbations that would violate the conservation law of eq. (1), but would preserve the structure of the equation. This is easily furnished by the following modification of the original evolution equation:

$$
u_{i}^{n+1}=u_{i}^{n}+\epsilon g\left(u_{i}^{n}\right)+\left(f\left(u_{i-1}^{n}\right)-2 f\left(u_{i}^{n}\right)+f\left(u_{i+1}^{n}\right)\right),
$$

where the local map function $f(x)$ is the same as above and a perturbation $g(x)$ is introduced. One can easily notice that in the simplest case of the uniform state it reduces to the equation determining the evolution of the average density,

$$
u^{n+1}=u^{n}+\epsilon g\left(u^{n}\right) .
$$

This equation, though, does not provide us with any reliable information concerning the dynamics of the average density in the case of a non-uniform state.

Second, in order to be able to compare two systems, one with and one without the conservation law, we should ensure that the latter is violated only "mildly." In other words, we would like the perturbed system to have a phase diagram that could be compared to that of the original system. In particular we would like to preserve the dimensionality of the parameter space. Since $u$ is no longer conserved it is not a parameter of the dynamics. Instead we introduce another parameter $u_{0}$ that will enter the evolution equation through the perturbation function $g(x)$.

By "mild" conservation violation we also mean that the change of the originally conserved average density $u$ during any single time-step is sufficiently small:

$$
\left|u^{n+1}-u^{n}\right| \ll 1 \text {. }
$$

We would also like the fluctuation of the average density $u$ to be bounded, such that

$$
u_{0}-\delta u<u^{n}<u_{0}+\delta u,
$$

for some finite $\delta u$ at any time step $n$. Then we would be able to compare the dynamics of the perturbed system with that of the original system with conserved quantity $u \approx u_{0}$.

Our primary interest in this section is the relation of the conservation law to the existence of a singularity in the spectrum of Lyapunov exponents. It is thus reasonable to compare the perturbed system with the original one, described by the equation (1), at the value of the conserved quantity $u=0.8$, where the conserving system displays a strongly chaotic dynamics and has a Lyapunov spectrum with a pronounced singularity at $\lambda=0$.

We will start with the following choice of the (nonlinear) perturbation function:
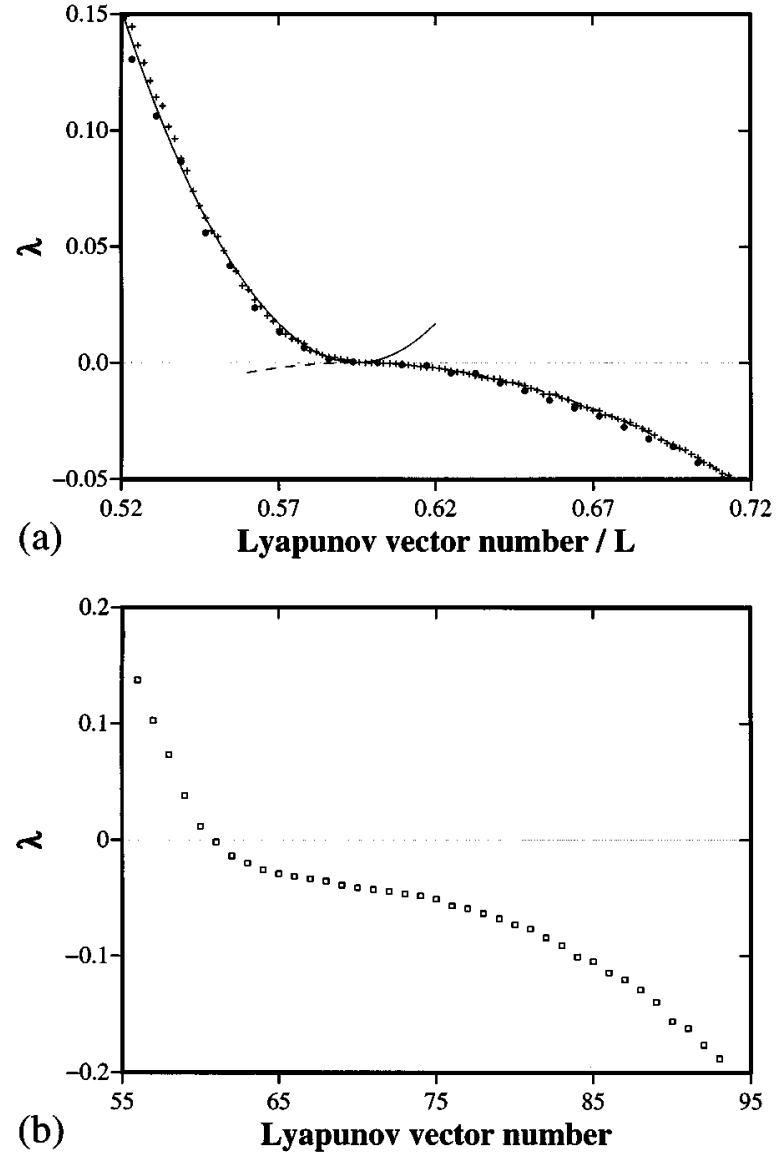

FIG. 14. "Slow" part of Lyapunov spectra of the unperturbed system and the system with nonlinear "dissipative" perturbation. (b) The spectrum of the perturbed system $\left(u_{0}=0.8, L=128\right)$ with $\epsilon=0.2$ appears to be shifted downwards and tilted with respect to (a) the spectrum of the unperturbed system $(u=0.8)$. Here the data for two lattice sizes, $L=128(\bullet)$ and $L=512(+)$, is superimposed to show the finite-size effects. The density of exponents is seen to be singular on both the positive and negative side. The solid and the dashed lines give the quadratic fit provided by eqs. (67) and (65), respectively.

$$
g(x)=\left(u_{0}-x\right)^{3},
$$

and choose $\epsilon=0.02$. The dynamics of the perturbed system seems qualitatively very similar to the dynamics of the unperturbed system, but now the average density $u$ is not conserved and fluctuates about $u_{0}$ with a standard deviation of order few percent.

Figure 14 of the Lyapunov exponent spectra focuses on the parts corresponding to slow evolution (small $\lambda$ ). Comparing the spectrum of the modified system with that of the original system for $u=u_{0}=0.8$, one can easily notice that the singularity is clearly present in the conserving case, while the spectrum of the perturbed system appears to be similar to the spectrum of the original system, but slightly tilted and shifted downwards.

In order to better understand the origin of such a metamorphosis it is advantageous to use another type of perturbation which is a lot easier to interpret and study analytically: 

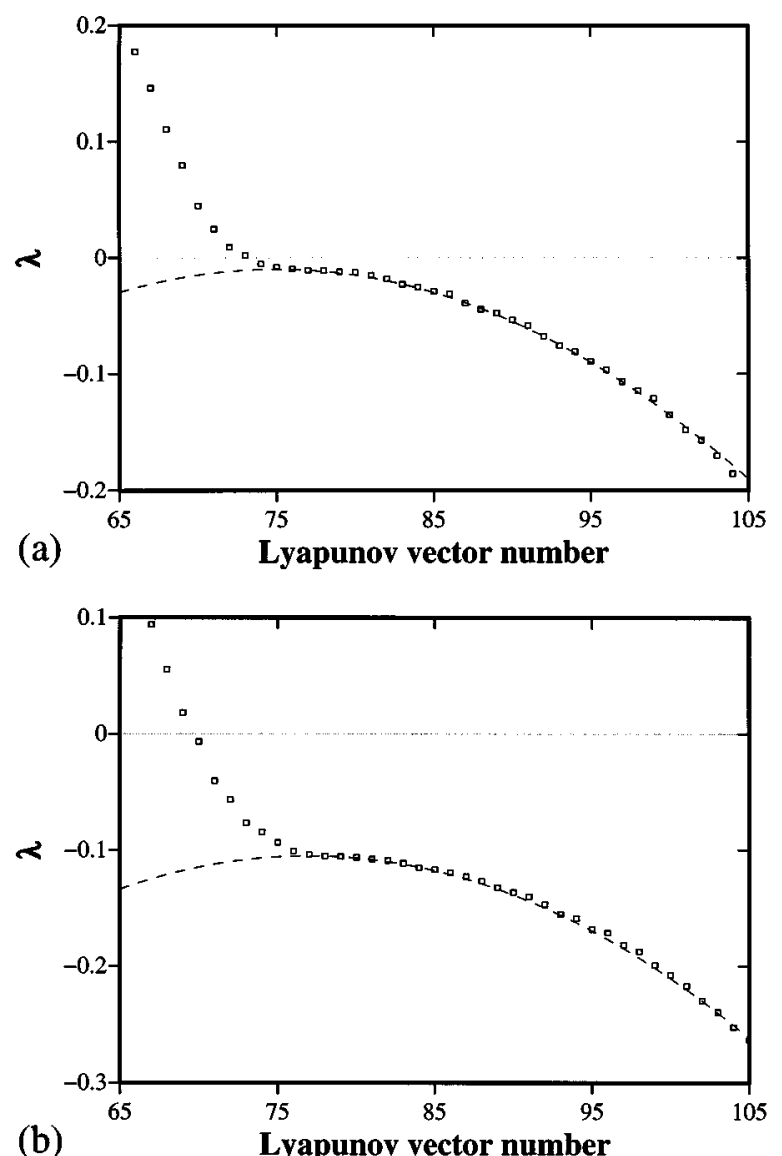

(b)

FIG. 15. "Slow" part of Lyapunov spectra of the system with linear "dissipative" perturbation. For small perturbation (a) $\epsilon=0.01$ as well as for large perturbation (b) $\epsilon=0.1$ the spectrum gets shifted downwards by $\delta \lambda \approx-\epsilon$ with respect to the spectrum of the unperturbed system. Lattice size is 128 and $u_{0}=0.8$. The dashed line gives the quadratic fit provided by eq. (77) with $D=0.33$ [assuming that $k$ is given by eq. (63) with $\alpha=0.5$ ].

$$
g(x)=u_{0}-x .
$$

Since $g(x)$ is linear, equation (71) now describes the evolution of the average density $u$ for an arbitrary initial state. For $\epsilon>0(0.001 \leqslant \epsilon \leqslant 0.1$ was used $)$ the asymptotic state is a configuration with $u=u_{0}$.

The effective Langevin equation corresponding to (75) should read as

$$
\partial_{t} u(x, t)=-\epsilon u+D \partial_{x}^{2} u+\partial_{x}^{2} \eta(x, t) .
$$

On large lengthscales noise averages out and we obtain the following dependence of the growth rate on the wave vector:

$$
\lambda(k)=-\epsilon-D k^{2},
$$

i.e. we should expect (for small $k$ 's) the Lyapunov spectrum of the perturbed system to be shifted downwards by $\delta \lambda=-\epsilon$ with respect to the spectrum of the conserving system, while retaining the same type of singularity. The total decay rate is then determined by a linear combination (at least for small enough coupling) of the diffusion with local dissipation.
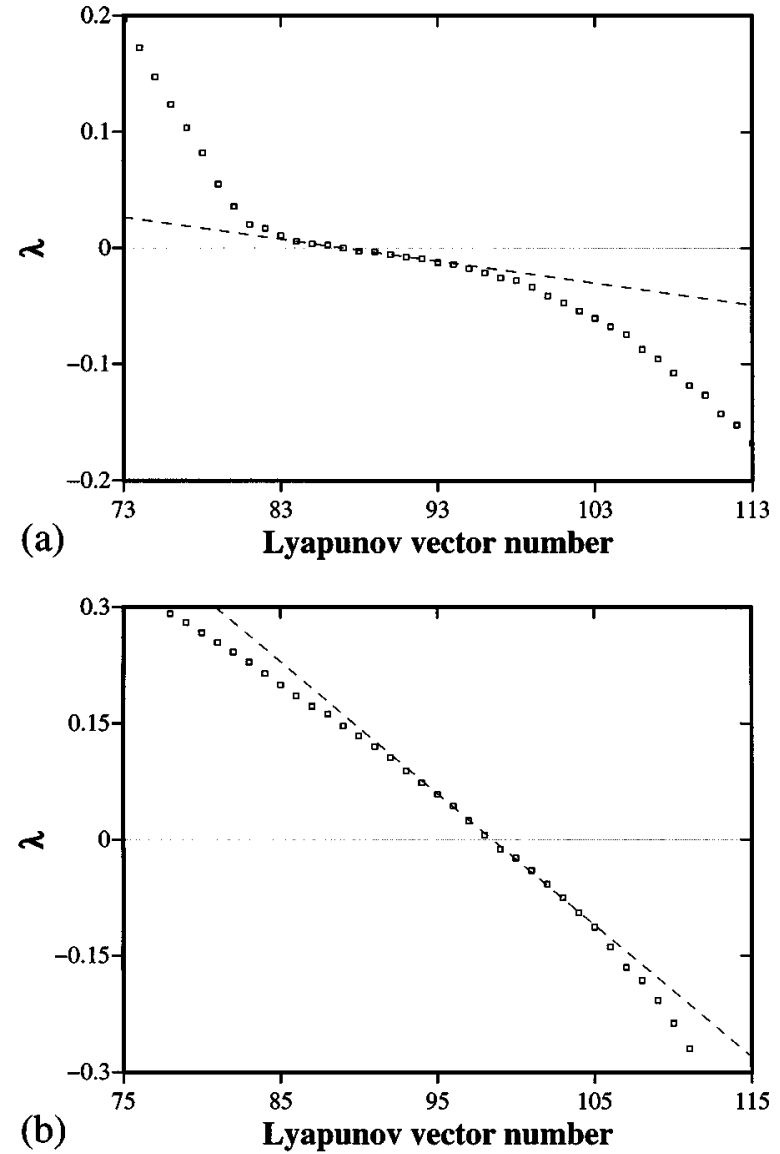

FIG. 16. "Slow" part of Lyapunov spectra of the system with "mixing", perturbation. For small perturbation (a) $\epsilon=0.1$ the tangent line (dashed) at the inflection becomes tilted. The singularity disappears. For very strong perturbation (b) $\epsilon=5.0$ the inflection vanishes completely. Lattice size is 128 and $u_{0}=0.8$.

Comparing the spectra of Lyapunov exponents of the modified system (fig. 15) and the original system [fig. 14(a)] for $u=u_{0}=0.8$ we see that the numerically obtained spectra of the perturbed system follow the prediction of the Langevin equation (76) quite precisely for small $(\epsilon=0.01)$ as well as for relatively strong $(\epsilon=0.1)$ perturbations.

We expect the negative shift of the "slow" part of the spectrum to be attributed to the dissipative nature of the perturbations used above. In fact we could have a positive shift or no shift at all. In order to see this we pick the non-linear perturbation function $g(x)$ with the first derivative which is not negative-definite:

$$
g(x)=\frac{\gamma^{2}\left(u_{0}-x\right)}{\left(\left(u_{0}-x\right)^{2}+\gamma^{2}\right)},
$$

with $\epsilon>0$. We used $\gamma^{2}=0.001$ and $0.01 \leqslant \epsilon \leqslant 5$.

Equation (71) does not hold anymore, but the numerical data suggests that the dynamics of the system is ergodic and the averaged density $u$ in the asymptotic state fluctuates about $u_{0}$ with fluctuations being again of order few percent.

Figure 16 shows the Lyapunov spectra of the perturbed system with $u_{0}=0.8$. This figure suggests that the spectrum is indeed shifted in the direction of positive rather than nega- 
tive values of $\lambda$. A small perturbation $(\epsilon=0.1)$ results in a small distortion of the original spectrum: the slope of the spectrum at the value $\lambda=0$ on both positive and negative sides becomes non-zero, i.e. the singularity in the density of Lyapunov exponents disappears. The slope increases with $\epsilon$ and for sufficiently strong perturbation $(\epsilon \gtrsim 2.0)$ all traces of the singularity vanish.

These examples suggest that there are, in fact, two different aspects of the singularity in the Lyapunov spectrum of a system with the conservation law. The first one is the presence of a singularity at some value $\lambda=\lambda_{0}$. The numerical data obtained suggests that the singularity survives in the special case of the linear perturbation function $g(x)$. A nonlinear perturbation results in the disappearance of the singularity. The Jacobian of a perturbed system can be written as

$\widetilde{J}_{k k^{\prime}}^{n}=J_{k k^{\prime}}^{n}+\epsilon \sum_{j \geqslant 1} \frac{g^{(j)}\left(u_{0}\right)}{(j-1) !} \prod_{i=1}^{j-1} \sum_{k_{i}} u_{k_{i}}^{n} \delta\left(\sum_{l=1}^{j-1} k_{l}-k+k^{\prime}\right)$,

where $J_{k k^{\prime}}^{n}$ is the Jacobian (56) of the conserving system. If $g(x)$ is non-linear the off-diagonal elements of $\widetilde{J}_{k k^{\prime}}^{n}$ become of order $O(1)$ instead of $O\left(k^{2}\right)$. As a result Fourier modes are no longer good as an approximation to the exact eigenvectors even for small $k$. The wave-vector $k$ can no longer label the slow modes of the system and therefore there is no reason to expect the singularity in the density of Lyapunov exponents to remain. It becomes "smoothed out" by the perturbation.

The second aspect is the actual value of $\lambda_{0}$ in case a singularity is present. We already saw that imposing linear perturbation (75) made $\lambda_{0}$ become negative [cf. (77)]. This is a consequence of the shift of the "slow" part of the spectrum as a whole in response to some local effects, e.g. dissipation.

We may therefore suggest, that since all known CML's with an additive conserved quantity possess a spectrum of Lyapunov exponents distinguished by the presence of the singularity at $\lambda=0$, a conservation law is a sufficient condition for the existence of such a singularity. It seems to be a necessary condition as well, at least in the class of models (1) studied in this paper. The singularity at $\lambda \neq 0$ indicates that there is a mechanism of (local) dissipation concurrent with diffusion and if this is eliminated the system becomes strictly conserving. This refinement might be helpful when looking for hidden conservation laws using Lyapunov spectra, ${ }^{9}$ numeric or experimental.

All three types of perturbation studied above are seen to have an effect on the phase diagram. For $\epsilon=0$ phase diagrams in three-dimensional parameter spaces $(a, b, u)$ and $\left(a, b, u_{0}\right)$ obviously coincide. Numerical data for all types of perturbation studied suggest that a gradual increase of the parameter $\epsilon$ makes the phase diagram of the perturbed system change continuously. The boundaries of the phases shift, making some of the phases shrink or completely disappear (e.g., sufficiently strong perturbation of any type obliterates the phase L2). Other phases may expand as the stability of their basic state is enhanced (as is the case for the phase L1 and the uniform state in the presence of a "dissipative" perturbation). We can say that, although the phase diagram is sensitive to the violations of the conservation law, it is robust with respect to sufficiently weak violations.

Since the changes in the phase diagram provoked by perturbations are found to be continuous, it is reasonable to expect that the phase transitions which were 1-order in the conserving system will remain 1-order after a perturbation is imposed. This leaves us an opportunity to observe how the characteristic exponents change with increasing perturbation. In our model the continuous phase transition at the boundary $\mathrm{T} 2-\mathrm{L} 1$ seems to be a promising point of investigation. Numerical data we have at the moment does not allow us to answer an important question of whether the universality classes change if the conservation law is violated, but we plan to return and investigate this later in more detail.

\section{CONCLUSIONS}

We have systematically investigated the properties of a coupled map lattice with dynamics constructed to satisfy a conservation law and to show spatiotemporal chaos.

The conserved quantity provides an additional control parameter: as its value is changed, a rich phase diagram with a number of phase transitions between ordered and disordered states is found. Both continuous and discontinuous transitions occur, as in coupled map lattices without a conservation law. The basic structure of the phase diagram is given by the linear stability boundaries of the ordered phases, although near the discontinuous transitions bistability may occur. Increasing the non-linearity, determined by the parameter $b$ [see fig.1(b)], renders the spatially uniform ordered phase (phase $L 1$ ) unstable. For $0<u<1 / 2$ the linear instability of the uniform state occurs via spatial period doubling (the zone boundary mode goes unstable). For $a<u<1 / 2$ the transition is immediately to a chaotic state, which takes the form of an increasing number of "turbulent" regions of roughly fixed size (defects) moving through the "laminar" background. This transition is continuous with a diverging correlation length and other scaling approaching the ordered state. For $0<u<a$ the transition to chaos is through a series of two subharmonic bifurcations, passing first to an intermediate 2-cycle state (the $L 2$ phase). The onset of chaos from $L 2$ is hysteretic. A complete subharmonic cascade is not observed. For $1 / 2<u<1$ the instability of the $L 1$ phase occurs through modes of all wave vectors going unstable together, and the appearance of chaos is also hysteretic with frozen chaotic domains developing.

As in thermodynamic systems, the phase transitions can be conveniently described by the use of order parameters, although since we are concerned with the growth of disorder the choice of the appropriate order parameter here is by no means obvious. In the case of the continuous transition we find that a number of proposed order parameters scale with distance to the transition point, thus allowing the evaluation of critical exponents, which may help to pin down whether universality classes for the onset of spatiotemporal chaos exist. An interesting question is whether the conservation law, 
which clearly affects the dynamic correlations, also changes the exponents and the universality class (if such a classification exists) of the phase transitions. The transition from $L 1$ to $T 2$ in the conserving model and in perturbed versions where the conservation law is weakly violated should provide a good arena for investigating this.

A symbolic description of the dynamics, reducing the complex states to regions of laminar (ordered) and turbulent (fluctuating) regions is useful in describing the chaotic states near the transitions. In particular we see that the onset of chaos always happens in the form of turbulent regions gradually spreading over the laminar background. Whenever the chaos appears to grow continuously, the turbulent fluctuations appear in the form of turbulent defects, usually propagating across the system, with size $l_{t}$ of order a few lattice spacings. Similar results were obtained by Kaneko ${ }^{12}$ for the non-conserving CML, although in our case there seems to be a stronger tendency for the defects to propagate with constant velocity.

We have also studied the Lyapunov eigenvalues and eigenvectors of the chaotic states. A conspicuous feature of conserving models is the singularity in the density of Lyapunov exponents around $\lambda=0$. There is growing evidence that the singularity is associated with the existence of Lyapunov eigenvectors that are labelled by the wave vector $\vec{k}$ for small $k$-the singularity is then the usual Van Hove singularity coming from mode counting and the assumption of a smooth spectrum at small $k$, i.e., $\lambda=\lambda_{0}-D_{\text {eff }} k^{2}$. This leads immediately to a $\left|\lambda-\lambda_{0}\right|^{-1 / 2}$ singularity in the density of exponents.

Some evidence for this idea comes from the work of Bohr et al. ${ }^{9}$ who studied some models where the Lyapunov spectrum can be exactly calculated, and in which the eigenvectors are trivially Fourier modes, since the Jacobian is independent of the dynamic variables. In these models the singularity can occur at non-zero $\lambda_{0}$, and the singularity may be to larger or smaller $\lambda$.

For the conserving (and non-trivial) maps we have studied, the labelling of the Lyapunov eigenvectors by the wave vector and the expression for the Lyapunov spectrum, with $\lambda_{0}=0$ and $D$ positive, is suggested by a hydrodynamic analysis, which associates the Lyapunov eigenvectors with the diffusively decaying modes given by a long wavelength Langevin description. The Fourier power spectrum of the Lyapunov eigenvectors provides support for this explanation: for small negative $\lambda$ the power spectrum corresponding to the $m$-th eigenvalue $\lambda_{m}$ is indeed strongly peaked around a small $k$ with $k \propto\left(m-m_{0}\right) 2 \pi / L$ with $\lambda_{m_{0}}=0$. Note however that the diffusion constant estimated from the Lyapunov spectrum may be significantly different from other estimates, and the proportionality constant $\alpha$ is greater than 0.5 which would be the value from simply counting the Fourier modes. Indeed, there is also a concentration of spectral power towards small $k$ on the positive $\lambda$ side, with a peak wave vector which again appears to scale linearly with $m_{0}-m$ for $m$ sufficiently close to $m_{0}$. Hence the long wavelength Fourier modes contribute appreciably to the Lyapunov vectors corresponding to small positive as well as negative exponents, which explains the deviation of $\alpha$ from 0.5 . There is no understanding from the hydrodynamic approach of the positive eigenvalue long wavelength modes and associated singularity in the density of exponents.

Adding the linear perturbation (75) makes $\lambda_{0}$ non-zero, but $\vec{k}$ remains a good label. Non-linear perturbations that eliminate the conservation law however destroy the singularity, and presumably in this case $\vec{k}$ is no longer a good label. Thus the conservation law appears to be a sufficient condition for Lyapunov spectrum singularity, but a complete quantitative understanding of this association remains lacking.

\section{ACKNOWLEDGMENTS}

R.O.G. would like to thank Professor N. R. Corngold for many helpful discussions. The authors gratefully acknowledge the support of the National Science Foundation under Grant No. DMR9013984.

${ }^{1}$ M. Bourzutschky and M. C. Cross, Chaos 2, 173 (1992).

${ }^{2}$ S. Puri, R. C. Desai, and R. Kapral, Physica D 50, 207 (1991).

${ }^{3}$ G. Grinstein, Y. He, C. Jayaprakash, and B. Bolker, Phys. Rev. A 44, 4923 (1991).

${ }^{4}$ Y. Kuramoto and T. Tsuzuki, Prog. Theor. Phys. 55, 356 (1976).

${ }^{5}$ J. W. Cahn and J. E. Hilliard, Trans. Metall. Soc. AIME 242, 166 (1968).

${ }^{6}$ N. B. Tufillaro, R. Ramshankar, and J. P. Gollub, Phys. Rev. Lett. 62, 422 (1989).

${ }^{7}$ S. Kai and W. Zimmermann, Prog. Theor. Phys. Suppl. 99, 458 (1989).

${ }^{8}$ M. Yamada and K. Okhitani, J. Phys. Soc. Jpn. 56, 4210 (1987).

${ }^{9}$ T. Bohr, G. Grinstein, and C. Jayaprakash, Chaos 5, 412 (1995).

${ }^{10}$ D. Ruelle, Commun. Math. Phys. 87, 287 (1982).

${ }^{11}$ J. P. Eckmann and D. Ruelle, Rev. Mod. Phys. 57, 617 (1985).

${ }^{12}$ K. Kaneko, Physica D 34, 1 (1989).

${ }^{13}$ J. Miller and D. A. Huse, Phys. Rev. E 48, 2528 (1993)

${ }^{14}$ P. Grassberger, Z. Phys. B 47, 365 (1982).

${ }^{15}$ K. Kaneko, Prog. Theor. Phys. 74, 1033 (1985).

${ }^{16}$ K. Kaneko, Physica D 23, 436 (1986).

${ }^{17}$ H. Chate and P. Manneville, Physica D 37, 33 (1989).

${ }^{18}$ P. Grassberger and T. Schreiber, Physica D 50, 177 (1991).

${ }^{19}$ H. Chate and P. Manneville, Physica D 32, 409 (1988).

${ }^{20} \mathrm{P}$. Manneville, Dissipative Structures and Weak Turbulence (Academic, San Diego, 1990)

${ }^{21}$ S. Ciliberto, Ann. Phys. 19, 715 (1994).

${ }^{22}$ S. Ciliberto and B. Nicolaenko, Europhys. Lett. 14, 303 (1991).

${ }^{23}$ P. Manneville, Lect. Notes Phys. 230, 319 (1985).

${ }^{24}$ H. Chate, Europhys. Lett. 21, 419 (1993).

${ }^{25}$ P. C. Hohenberg and B. I. Halperin, Rev. Mod. Phys. 49, 435 (1977). 